\title{
STRAIN FIELD IN A SEMI-INFINITE MEDIUM DUE TO AN INCLINED RECTANGULAR FAULT
}

\author{
Takaya IWASAKI and Ryosuke SATo \\ Geophysical Institute, Faculty of Science, University of Tokyo, Tokyo, Japan
}

(Received November 4, 1978)

Analytical expressions are derived for the strain fields at an arbitrary depth due to an inclined fault in a semi-infinite medium. These expressions, being composed of elementary functions, are suitable for numerical computations. Basing upon these expressions, we develop a computer program, by which contour maps of deformation fields can be drawn in an arbitrary area designated by input parameters.

Using this program, several examples of the strain fields in a semi-infinite medium are shown. Effect of the free surface is clarified by comparing patterns for a semi-infinite medium with those for an infinite medium. For example, in the vertical cross sections, this effect yields saddle points and concentration of contour lines and also the "reverse area", where sense of the deformation is opposite to that for the case of an infinite medium. The reverse area appears clearly in the shear strain components given by differentiating horizontal displacements with respect to depth.

\section{Introduction}

Recently geodetic data as well as seismic wave data have been playing important roles in explaining focal process and determining focal parameters.

Since the introduction of "the elasticity theory of dislocations" (STEKETEE, 1958a, b) to seismology, theoretical studies on the crustal deformation have been done by many investigators. For the displacement field, Mansinia and SMyine (1971), following Savage and Hastie (1966), derived analytical expressions at an arbitrary depth due to an inclined fault in a semiinfinite Poisson solid. Theoretical strain fields were first investigated only for some special cases by several authors (Chinnery, 1963; Press, 1965; Canitez and ToKsöz, 1972). SATo and MATsu'Ura (1974) first obtained simple analytical expressions for strain fields on the free surface due to an inclined rectangular fault.

However analytical representations for strain or stress fields at an arbitrary depth have not been given in perfect forms as yet. For example, CHINNERY (1963) showed patterns of stress components in a semi-infinite medium only for a vertical strike-slip fault. ALWINE (1974) derived repre- 
sentations for the strain field from direct differentiation of Mansinha and Smylie's results. In his results, however, the strain components given by derivatives of displacements with respect to depth are not included and his formulations are too complicated to grasp the physical meaning.

In this paper, general analytical expressions are given for all the strain components at an arbitrary depth due to an inclined fault in a semi-infinite medium. For this purpose, we proceed as follows;

1. Derivation of expressions for the displacement field due to a point source in a semi-infinite medium.

2. Analytical double integration over the fault plane of the expressions given in Procedure 1.

Going through Procedure 1, not only the physical meaning of the expressions for the strain fields can be clarified, but also the labor for the theoretical calculation in Procedure 2 can be reduced, as described in the next section.

\section{Analytical Representation of Strain Field}

\subsection{Static displacements due to a point source}

We take the Cartesian coordinate system $(x, y, z)$ where the half space occupies the region $z<0$. When a point source is located at $\left(0,0,-d_{0}\right)$, static displacements at an arbitrary depth are given, under the boundary conditions that the stress components $\tau_{z x}, \tau_{z y}$ and $\tau_{z z}$ vanish on the free surface, by

$$
\begin{aligned}
& u=\left(u_{x}, u_{y}, u_{z}\right)=\sum_{i=1}^{5} u^{i} \\
& u_{x}{ }^{1}=- \frac{U_{0}}{4 \pi}\left[\alpha \frac{3 x p_{1} q_{1}}{R_{1}{ }^{\mathrm{b}}} \sin \lambda+\left\{(1-\alpha) \frac{q_{1}}{R_{1}{ }^{3}}+\alpha \frac{3 x^{2} q_{1}}{R_{1}{ }^{5}}\right\} \cos \lambda\right], \\
& u_{y}{ }^{1}=-\frac{U_{0}}{4 \pi}\left[\left\{(1-\alpha) \frac{1}{R_{1}{ }^{3}}\left(p_{1} \sin \delta+q_{1} \cos \delta\right)+\alpha \frac{3 y p_{1} q_{1}}{R_{1}{ }^{5}}\right\} \sin \lambda\right. \\
&\left.+\left\{(1-\alpha) \frac{x}{R_{1}{ }^{3}} \sin \delta+\alpha \frac{3 x y q_{1}}{R_{1}{ }^{5}}\right\} \cos \lambda\right], \\
& u_{z}{ }^{1}=-\frac{U_{0}}{4 \pi}\left[\left\{(1-\alpha) \frac{1}{R_{1}{ }^{3}}\left(p_{1} \cos \delta-q_{1} \sin \delta\right)-\alpha \frac{3 d_{1} p_{1} q_{1}}{R_{1}{ }^{\mathrm{b}}}\right\} \sin \lambda\right. \\
&\left.+\left\{(1-\alpha) \frac{x}{R_{1}{ }^{3}} \cos \delta-\alpha \frac{3 x d_{1} q_{1}}{R_{1}{ }^{5}}\right\} \cos \lambda\right],
\end{aligned}
$$

where

$$
\begin{gathered}
d_{1}=d_{0}+z, \\
p_{1}=y \cos \delta+d_{1} \sin \delta, \quad q_{1}=y \sin \delta-d_{1} \cos \delta, \\
R_{1}=\left(r^{2}+d_{1}^{2}\right)^{1 / 2}=\left(x^{2}+y^{2}+d_{1}^{2}\right)^{1 / 2},
\end{gathered}
$$


where

$$
\left.\begin{array}{c}
\alpha=1-\left(V_{\mathrm{s}} / V_{\mathrm{P}}\right)^{2} . \\
u_{x}{ }^{2}=-u_{x}{ }^{1}(d, p, q, R), \\
u_{y}{ }^{2}=-u_{y}{ }^{1}(d, p, q, R), \\
u_{z}{ }^{2}=-u_{z}{ }^{1}(d, p, q, R),
\end{array}\right\}
$$

$$
d=d_{0}-z
$$

$$
\begin{aligned}
& p=y \cos \delta+d \sin \delta, \quad q=y \sin \delta-d \cos \delta, \\
& R=\left(r^{2}+d^{2}\right)^{1 / 2} .
\end{aligned}
$$

$\boldsymbol{u}^{1}(d, p, q, R)$ is the displacements for which $d_{1}, p_{1}, q_{1}$ and $R_{1}$ in Eq. (2.2) are replaced by $d, p, q$ and $R$ respectively.

$$
\begin{aligned}
& u_{x}^{3}=-\frac{U_{0}}{2 \pi}\left[\left\{\begin{array}{c}
3 x p q \\
R^{6}
\end{array}-\frac{\partial F_{1}}{\partial x} \sin \delta \cos \delta\right\} \sin \lambda+\left\{\frac{3 x^{2} q}{R^{5}}+\frac{\partial F_{2}}{\partial x} \sin \delta\right\} \cos \lambda\right], \\
& u_{y}=-\frac{U_{0}}{2 \pi}\left[\left\{\frac{3 y p q}{R^{b}}-\frac{\partial F_{1}}{\partial y} \sin \delta \cos \delta\right\} \sin \lambda+\left\{\frac{3 x y q}{R^{6}}+\frac{\partial F_{2}}{\partial y} \sin \delta\right\} \cos \lambda\right] \text {, } \\
& \left.u_{z}^{3}=-\frac{U_{0}}{2 \pi}\left[\left\{\frac{3 d_{0} p q}{R^{0}}-\frac{\partial F_{3}}{\partial x} \sin \delta \cos \delta\right\} \sin \lambda+\left\{\frac{3 x d_{0} q}{R^{5}}+\frac{\partial F_{3}}{\partial y} \sin \delta\right\} \cos \lambda\right],\right)
\end{aligned}
$$

where

$$
\begin{aligned}
& F_{1}=\frac{1-\alpha}{\alpha}\left\{\frac{x^{2}-y^{2}}{r^{4}}(R-d)-\frac{x^{2}}{r^{2} R}\right\}, \\
& F_{2}=\frac{1-\alpha}{\alpha}\left\{\frac{2 x y}{r^{4}}(R-d)-\frac{x y}{r^{2} R}\right\}, \\
& F_{3}=\frac{1-\alpha}{\alpha} \frac{x}{r^{2}}\left(1-\frac{d}{R}\right) .
\end{aligned}
$$

$u_{x}^{4}=\frac{U_{0}}{2 \pi}(1-\alpha) z\left[\begin{array}{l}3 x \\ R^{0}\end{array}(p \cos \delta-q \sin \delta) \sin \lambda-\frac{1}{R^{5}}\left(R^{2}-3 x^{2}\right) \cos \delta \cos \lambda\right]$,

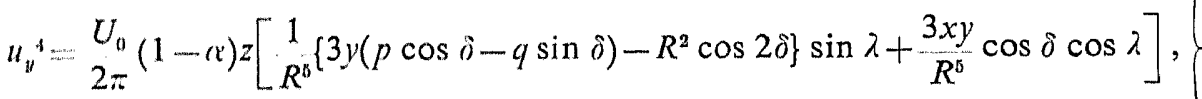

$u_{z}^{4}=\frac{U_{0}}{2 \pi}(1-\alpha) z\left[\frac{1}{R^{6}}\left(R^{2}-3 x^{2}\right) \sin \delta \cos \delta \sin \lambda+\frac{3 x y}{R^{6}} \sin \delta \cos \lambda\right]$,

$u_{x}^{3}=-\frac{3 U_{0}}{2 \pi} \alpha z d_{n}\left[\begin{array}{c}5 x p q \\ R^{7}\end{array} \sin \lambda+\frac{q}{R^{7}}\left(5 x^{2}-R^{2}\right) \cos \lambda\right]$

$u_{y}^{3}=-\frac{3 U_{0}}{2 \pi} \alpha z d_{0}\left[\frac{1}{R^{7}}\left\{5 y p q-R^{2}(p \sin \delta+q \cos \delta)\right\} \sin \lambda\right.$

$\left.+\frac{x}{R^{7}}\left(5 y q-R^{2} \sin \delta\right) \cos \lambda\right]$, 


$$
\begin{aligned}
u_{z}^{5}= & \frac{3 U_{0}}{2 \pi} \alpha z d_{0}\left[\frac{1}{R^{7}}\left\{5 d p q+R^{2}(p \cos \delta-q \sin \delta)\right\} \sin \lambda\right. \\
& \left.+\frac{x}{R^{7}}\left(5 d q+R^{2} \cos \delta\right) \cos \lambda\right] .
\end{aligned}
$$

In the above expressions, $U_{0}$ is the amount of displacement discontinuity and $V_{\mathrm{P}}$ and $V_{\mathrm{s}}$ are $\mathrm{P}$ and $\mathrm{S}$ wave velocities. Other parameters, $\delta$ and $\lambda$ are defined as shown in Fig. 1.

$u^{1}$ represents the displacement vector due to a point source at $\mathrm{S}\left(0,0,-d_{0}\right)$ in an infinite medium (Fig. 2). $\boldsymbol{u}^{2}$ has the same form as the displacement vector due to an image source at $S^{\prime}\left(0,0, d_{0}\right)$. Figure 2 illustrates the variables in Eqs. (2.3), (2.4), (2.8), and (2.9). $\boldsymbol{u} \boldsymbol{u}^{4}$ and $\boldsymbol{u}^{5}$, which are proportional to the depth, $z$, of an observing point, are necessary only when the deformation within the medium is considered. For an observing point at the surface, $\boldsymbol{u}^{1}$ and $\boldsymbol{u}^{2}$ cancel each other and $\boldsymbol{u}^{4}$ and $\boldsymbol{u}^{5}$ vanish. Hence, only $\boldsymbol{u}^{3}$ exists and the displacement field is reduced to SATo and MATSU'URA's expressions (1974).

\subsection{Integration over the fault plane}

Strain components concerned with $\boldsymbol{u}^{1}, \boldsymbol{u}^{2}$ and $\boldsymbol{u}^{3}$ have already been obtained by Sato and Matsu'ura except for the components derived from $\boldsymbol{u}^{3}$ by differentiating with respect to depth and therefore most of our effort for the theoretical calculation is restricted to the integration of $\boldsymbol{u}^{4}$ and $\boldsymbol{u}^{5}$.

For a fault with length $2 L$ and width $w$ (Fig. 1), the displacement fields can be obtained by replacing $x, y$ and $d_{0}$ by $x-\xi, y-\eta \cos \delta$ and $d_{0}-\eta \sin \delta$ in Eqs. (2.1) (2.14) and then applying an integration operator

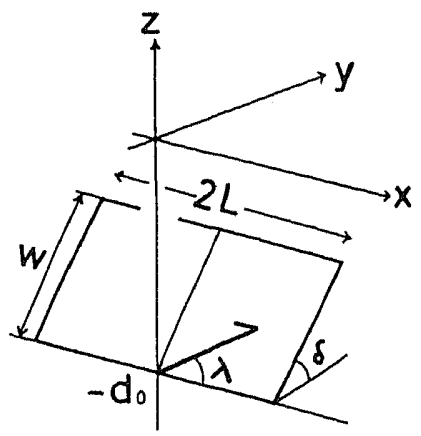

Fig. 1. Coordinates and geometry of the fault model. $w$, fault width; $2 L$, fault length; $\delta$, dip-angle; $\lambda$, slip-angle; $d_{0}$, fault depth.

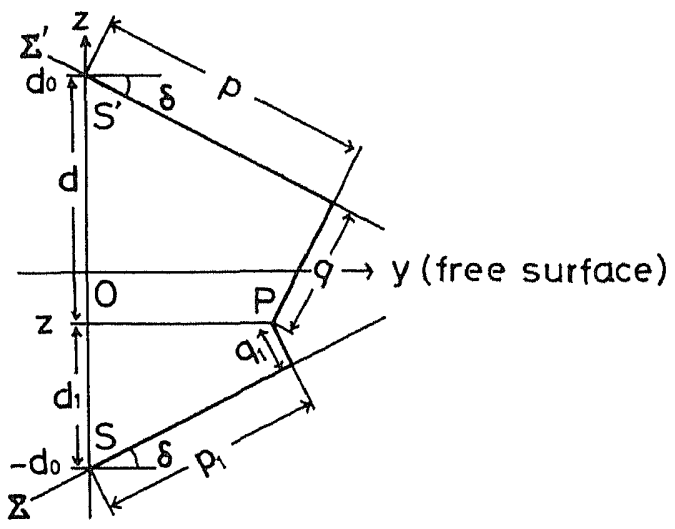

Fig. 2. Geometrical meaning of variables in Eqs. (2.3), (2.4), (2.8), and (2.9). In this figure, $q_{1}$ and $q$ are negative. $\Sigma$, fault plane; $\Sigma$, image fault plane; $\delta$, dip-angle. 


$$
\int_{-L}^{L} d \xi \int_{0}^{w} d \eta
$$

Introducing new variables $\xi^{\prime}, \eta^{\prime \prime}$ for $\boldsymbol{u}^{1}$ and $\xi^{\prime}, \eta^{\prime}$ for $\boldsymbol{u}^{2} \sim \boldsymbol{u}^{5}$ in place of $\xi, \eta$ defined by

$$
\left.\begin{array}{c}
\xi^{\prime}=x-\xi, \\
\eta^{\prime}=p-\eta, \\
\gamma^{\prime \prime}=p_{1}-\eta,
\end{array}\right\}
$$

the integration operator $(2.15)$ can be written as

for $\boldsymbol{u}^{1}$, and

$$
\left.\begin{array}{c}
\int_{x-L}^{x+L} d \xi^{\prime} \int_{p_{1}-w}^{p_{1}} d \eta^{\prime \prime}, \\
y-\eta \cos \delta=\eta^{\prime \prime} \cos \delta+q_{1} \sin \delta, \\
d_{1}-\eta \sin \delta=\eta^{\prime \prime} \sin \delta-q_{1} \cos \delta, \\
R_{1}=\left(\xi^{\prime 2}+\eta^{\prime \prime 2}+q_{1}^{2}\right)^{1 / 2},
\end{array}\right\}
$$

$$
\left.\begin{array}{c}
\int_{x-L}^{x+L} d \xi^{\prime} \int_{\eta-w}^{p} d \eta^{\prime}, \\
y-\eta \cos \delta=\eta^{\prime} \cos \delta+q \sin \delta, \\
d-\eta \sin \delta=\eta^{\prime} \sin \delta-q \cos \delta, \\
R=\left(\xi^{\prime 2}+\eta^{\prime 2}+q^{2}\right)^{1 / 2},
\end{array}\right\}
$$

for $\boldsymbol{u}^{2} \sim \boldsymbol{u}^{5}$. It is not so difficult to perform the integration of derivatives of displacements with respect to $x, y$ and $z$ and the final results in indefinite integral forms are given by

$$
\left.\begin{array}{lll}
\partial u_{x}=\sum_{i=1}^{5} U_{x x}{ }^{i}, & \frac{\partial u_{x}}{\partial y}=\sum_{i=1}^{5} U_{x y}{ }^{i}, & \frac{\partial u_{x}}{\partial z}=\sum_{i=1}^{5} U_{x z}{ }^{i} \\
\partial u_{y}=\sum_{i=1}^{5} U_{y x}{ }^{i}, & \frac{\partial u_{y}}{\partial y}=\sum_{i=1}^{5} U_{y y}{ }^{i}, & \frac{\partial u_{y}}{\partial z}=\sum_{i=1}^{5} U_{y z}{ }^{i} \\
\partial u_{z}=\sum_{i=1}^{5} U_{z x}{ }^{i}, & \frac{\partial u_{z}}{\partial y}=\sum_{i=1}^{5} U_{z y}{ }^{i}, & \frac{\partial u_{z}}{\partial z}=\sum_{i=1}^{5} U_{z z}{ }^{i} \cdot
\end{array}\right\}
$$

The superscript $i$ of $U_{\alpha \beta}{ }^{i}$ indicates that $U_{\alpha \hat{\beta}}{ }^{i}$ is derived from $u^{i}$ in Eq. (2.1). $U_{r i i^{i}}{ }^{i}$ is given in the following;

$$
\begin{aligned}
& U_{x x z}{ }^{2}=-\frac{U_{0}}{4 \pi}\left[\alpha P_{x x}+(1-\alpha) \frac{q}{R\left(R+\eta^{\prime}\right)} \cos \lambda\right], \\
& U_{x y}{ }^{2}=U_{0}\left[\alpha P_{x y}+(1-\alpha)\left\{\frac{\xi^{\prime}}{R\left(R+\eta^{\prime}\right)} \sin \delta+\frac{D}{R\left(R+\xi^{\prime}\right)}\right\} \cos \lambda\right], \\
& U_{x z}{ }^{2}=\frac{U_{0}}{4 \pi}\left[\alpha P_{x z}+(1-\alpha)\left\{\frac{\xi^{\prime}}{R\left(R+\eta^{\prime}\right)} \cos \delta+\frac{Y_{1}}{R\left(R+\xi^{\prime}\right)}\right\} \cos \lambda\right],
\end{aligned}
$$




$$
\begin{aligned}
& U_{y x}{ }^{2}=-\frac{U_{0}}{4 \pi}\left[\alpha P_{y x}+(1-\alpha)\left\{\frac{1}{R} \sin \delta+\frac{q}{R\left(R+\eta^{\prime}\right)} \cos \delta\right\} \sin \lambda\right. \\
& \left.+(1-\alpha) \frac{\xi^{\prime}}{R\left(R+\eta^{\prime}\right)} \sin \delta \cos \lambda\right] \text {, } \\
& U_{y y}{ }^{2}=\frac{U_{0}}{4 \pi}\left[\alpha P_{y y}+(1-\alpha)\left\{\frac{\xi^{\prime}}{2 R\left(R+\eta^{\prime}\right)} \sin 2 \delta-\frac{q}{R\left(R+\xi^{\prime}\right)}\right\} \sin \lambda\right. \\
& \left.-(1-\alpha) \frac{R \cos \delta+Y_{1}}{R\left(R+\eta^{\prime}\right)} \sin \delta \cos \lambda\right] \\
& U_{y z}{ }^{2}=\frac{U_{0}}{4 \pi}\left[\alpha P_{y z}+(1-\alpha)\left\{\frac{\eta^{\prime}}{R\left(R+\xi^{\prime}\right)}+\frac{\xi^{\prime}}{2 R\left(R+\eta^{\prime}\right)}\right.\right. \\
& \left.\left.\left.+\frac{\xi^{\prime}}{2 R\left(R+\eta^{\prime}\right)} \cos 2 \delta\right\} \sin \lambda+\frac{R \sin \delta+D}{R\left(R+\eta^{\prime}\right)} \sin \delta \cos \lambda\right], \quad\right) \\
& U_{z x}^{2}=\frac{U_{0}}{4 \pi}\left[\alpha P_{z x}+(1-\alpha)\left\{\frac{1}{R} \cos \delta-\frac{q}{R\left(R+\eta^{\prime}\right)} \sin \delta\right\} \sin \lambda\right. \\
& \left.+(1-\alpha) \frac{\xi^{\prime}}{R\left(R+\eta^{\prime}\right)} \cos \delta \cos \lambda\right] \text {, } \\
& U_{z y}{ }^{2}=\frac{U_{0}}{4 \pi}\left[\alpha P_{z y}+(1-\alpha)\left\{\frac{\eta^{\prime}}{R\left(R+\xi^{\prime}\right)}+\frac{\xi^{\prime}}{2 R\left(R+\eta^{\prime}\right)}-\frac{\xi^{\prime}}{2 R\left(R+\eta^{\prime}\right)} \cos 2 \delta\right\} \sin \lambda\right. \\
& \left.+(1-\alpha) \frac{R \cos \delta+Y_{1}}{R\left(R+\eta^{\prime}\right)} \cos \delta \cos \lambda\right] \text {, } \\
& U_{z z}{ }^{2}=\frac{U_{0}}{4 \pi}\left[\alpha P_{z z}-\alpha\left(\frac{\xi^{\prime} q}{2 R^{3}} \sin \lambda+\frac{1}{2} q \xi^{\prime 2} A_{\eta^{\prime}} \cos \lambda\right)+(1-\alpha)\left\{\begin{array}{c}
\xi^{\prime} \\
2 R\left(R+\eta^{\prime}\right)
\end{array} \sin 2 j\right.\right. \\
& \left.\left.+\frac{q}{R\left(R+\xi^{\prime}\right)}\right\} \sin \lambda-(1-\alpha) \frac{R \sin \delta+D}{R\left(R+\eta^{\prime}\right)} \cos \delta \cos \lambda\right] \text {, } \\
& U_{x x}{ }^{1}=-U_{x x}{ }^{2}\left(\eta^{\prime \prime}, p_{1}, q_{1}, R_{1}\right), \quad U_{x y}{ }^{1}=-U_{x y}{ }^{2}\left(\gamma^{\prime \prime}, p_{1}, q_{1}, R_{1}\right), \\
& U_{x z}{ }^{1}=U_{x z}{ }^{2}\left(\eta^{\prime \prime}, p_{1}, q_{1}, R_{1}\right) \text {, } \\
& U_{y x}{ }^{1}=-U_{y x}{ }^{2}\left(\eta^{\prime \prime}, p_{1}, q_{1}, R_{1}\right), \quad U_{y y}{ }^{1}=-U_{y y}{ }^{2}\left(\eta^{\prime \prime}, p_{1}, q_{1}, R_{1}\right), \\
& U_{y z}{ }^{3}=U_{y z}{ }^{2}\left(\eta^{\prime \prime}, p_{1}, q_{1}, R_{1}\right) \text {, } \\
& U_{z x}{ }^{1}=-U_{z x}{ }^{2}\left(\eta^{\prime \prime}, p_{1}, q_{1}, R_{1}\right), \quad U_{z y}{ }^{1}=-U_{z y}{ }^{2}\left(\gamma^{\prime \prime}, p_{1}, q_{1}, R_{1}\right) \text {, } \\
& U_{z z}{ }^{1}=U_{z z}{ }^{2}\left(\eta^{\prime \prime}, p_{1}, q_{1}, R_{1}\right) \text {. }
\end{aligned}
$$

$U_{\alpha \beta}{ }^{2}\left(\eta^{\prime \prime}, p_{1}, q_{1}, R_{1}\right)$ indicates the expression that $\gamma^{\prime}, p, q$ and $R$ in $U_{\alpha \beta}{ }^{2}$ are replaced by $\eta^{\prime \prime}, p_{1}, q_{1}$ and $R_{1}$.

$$
U_{x x}^{3}=\frac{U_{0}}{2 \pi}\left[P_{x x}+I_{1} \sin \delta \cos \delta \sin \lambda-I_{2} \sin \delta \cos \lambda\right],
$$


Strain Field in a Semi-Infinite Medium Due to an Inclined Rectangular Fault

$\left.\begin{array}{l}U_{x y}{ }^{3}=-\frac{U_{0}}{2 \pi}\left[P_{x y y}-I_{2} \sin \delta \cos \delta \sin \lambda-\left\{\begin{array}{cc}1-\alpha & \xi^{\prime} \\ \alpha^{\prime} & R\left(R+\gamma^{\prime}\right)\end{array}+I_{1}\right\} \sin \delta \cos \lambda\right], \\ U_{x z}{ }^{3}=-U_{0}\left[P_{x z}+I_{3} \sin \delta \cos \delta \sin \lambda-I_{4} \sin \delta \cos \lambda\right],\end{array}\right\}$

$U_{y x}{ }^{3}=\frac{U_{0}}{2 \pi}\left[P_{y, x}+I_{2} \sin \delta \cos \delta \sin \lambda+\left\{\begin{array}{cc}1-\alpha & \xi^{\prime} \\ \alpha & R\left(R+\gamma^{\prime}\right)\end{array}+I_{1}\right\} \sin \delta \cos \lambda\right]$, $U_{u y}{ }^{3}=-U_{0}\left[P_{u y}+\left\{\begin{array}{cc}1-\alpha^{\prime} & \xi^{\prime} \\ \alpha & R\left(R+\gamma^{\prime}\right)\end{array}+I_{1}\right\} \sin \delta \cos \delta \sin \lambda\right.$

$\left.-\left\{\begin{array}{cc}1-\alpha & 1 \\ \alpha & R\end{array}\left(\begin{array}{c}q \\ R+\eta^{\prime}\end{array} \sin \delta+\cos \delta\right)+I_{2}\right\} \sin \delta \cos \lambda\right]$,

$U_{y z}{ }^{3}=-\frac{U_{0}}{2 \pi}\left[P_{n z}+I_{4} \sin \delta \cos \delta \sin \lambda\right.$

$\left.-\left\{\begin{array}{cc}1-\alpha^{\prime} & 1 \\ \alpha & R\end{array}\left(\begin{array}{c}q \\ R+y^{\prime}\end{array} \cos \delta-\sin \delta\right)-I_{3}\right\} \sin i \cos \lambda\right]$,

$U_{z x}^{3}=-\frac{U_{10}}{2 \pi}\left[P_{z, r}-\left(\begin{array}{l}z q \\ R^{3}\end{array}+I_{3} \sin i \cos \delta\right) \sin \lambda-\left(\xi^{\prime} q z A_{\eta^{\prime}}-I_{4} \sin \delta\right) \cos \lambda\right]$,

$U_{z y^{3}}{ }^{3}=-\frac{U_{10}}{2 \pi}\left[P_{z y}-\left\{z\left(q Y_{1} A_{\xi^{\prime}}-\frac{1}{R\left(R+\xi^{\prime}\right)} \sin \delta\right)+I_{4} \sin \delta \cos \delta\right\} \sin \lambda\right.$

$+\left\{z\left(B_{r^{\prime}} \cdot \sin \delta-\frac{q}{R^{3}} \cos \delta\right)\right.$

$\left.\left.+\underset{r}{1-\alpha} \frac{1}{R}\left(\begin{array}{c}q \\ R+\gamma^{\prime}\end{array} \cos \delta-\sin \delta\right) \sin \delta-I_{3} \sin \delta\right\} \cos \lambda\right]$,

$U_{z z^{3}}=-\frac{U_{11}}{2 \pi}\left[P_{z z}-\left\{q\left(\begin{array}{c}\xi^{\prime} \\ 2 R^{3}\end{array}-\begin{array}{c}1 \\ R\left(R+\xi^{\prime}\right)\end{array}\right)-z\left(q D A_{\xi^{\prime}}+\frac{1}{R\left(R+\xi^{\prime}\right)} \cos \delta\right)\right.\right.$

$\left.-\begin{array}{cc}1-\alpha & \xi^{\prime} \\ \alpha & R\left(R+\gamma^{\prime}\right)\end{array} \sin i \cos \delta\right\} \sin \lambda+\left\{\begin{array}{l}1 \\ 2\end{array}\left(\begin{array}{c}\gamma^{\prime} q \\ R^{3}\end{array}+q^{3} A_{i j^{\prime}}\right)\right.$

$\left.\left.+z\left(B_{y^{\prime}} \cos i+\frac{q}{R^{3}} \sin \delta\right)-\alpha^{1-\alpha} \quad \frac{1}{\alpha^{\prime}}\left(\begin{array}{c}q \\ R+\gamma^{\prime}\end{array} \sin \delta+\cos \delta\right)\right\} \cos \lambda\right]$,

$$
\begin{aligned}
U_{x x}= & \frac{U_{0}}{2 \pi}(1-\alpha) z \xi^{\prime}\left[q A_{z^{\prime}} \sin \delta-\frac{1}{R^{3}} \cos \delta\right] \sin \lambda \\
& +\frac{U_{0}}{2 \pi}(1-\alpha) z\left[\begin{array}{c}
1 \\
R\left(R+\gamma^{\prime}\right)
\end{array}-\xi^{\prime 2} A_{\gamma^{\prime}}\right] \cos \delta \cos \lambda,
\end{aligned}
$$




$$
\begin{aligned}
& U_{x y}{ }^{4}=-\frac{U_{0}}{4 \pi}(1-\alpha) z\left[\frac{\eta^{\prime}}{R^{3}}+B_{r^{\prime}}+\left(\frac{\eta^{\prime}}{R^{3}}-B_{\eta^{\prime}}\right) \cos 2 \delta\right] \sin \lambda \\
& -\frac{U_{0}}{4 \pi}(1-\alpha) z \xi^{\prime}\left[\frac{1}{R^{3}}+q A_{\eta^{\prime}} \sin 2 \delta+\frac{1}{R^{3}} \cos 2 \delta\right] \cos \lambda, \\
& U_{x z}^{4}=V_{x}^{4}+\frac{U_{0}}{4 \pi}(1-\alpha) z\left[-\frac{2 q}{R^{3}}+\left(\frac{\eta^{\prime}}{R^{3}}-B_{\eta^{\prime}}\right) \sin 2 \delta\right] \sin \lambda \\
& +\frac{U_{0}}{4 \pi}(1-\alpha) z \xi^{\prime}\left[-q A_{\eta^{\prime}}+\frac{1}{R^{3}} \sin 2 \hat{\partial}-q A_{\eta^{\prime}} \cos 2 \delta\right] \cos \lambda, \\
& U_{y x}^{4}=U_{x y}^{4} \\
& U_{y y}{ }^{4}=\frac{U_{0}}{2 \pi}(1-\alpha) z\left[\left\{\left(q^{2}-Y_{1}^{2}\right) A_{\xi^{\prime}}+B_{\xi}, \cos 2 \delta\right\} \cos \delta\right. \\
& \left.+\frac{q}{2}\left\{-\xi^{\prime} A_{\eta^{\prime}}+\left(\xi^{\prime} A_{\eta^{\prime}}+2 \eta^{\prime} A_{\xi^{\prime}}\right) \cos 2 \delta\right\} \sin \delta\right] \sin \lambda \\
& -\frac{U_{0}}{4 \pi}(1-\alpha) z\left[\left(\frac{\eta^{\prime}}{R^{3}}-B_{\eta^{\prime}}\right)+\left(\frac{\eta^{\prime}}{R^{3}}+B_{\eta^{\prime}}\right) \cos 2 \delta+\frac{2 q}{R^{3}} \sin 2 \delta\right] \cos \delta \cos \lambda, \\
& U_{y z}^{4}=V_{y}^{4}+\frac{U_{0}}{2 \pi}(1-\alpha) z\left[Y_{1} D A_{\xi^{\prime}} \cos \delta\right. \\
& \left.-\left\{B_{\xi^{\prime}} \cos 2 \delta+\frac{q}{2}\left(\xi^{\prime} A_{\eta^{\prime}}+2 \eta^{\prime} A_{\xi^{\prime}}\right) \sin 2 \delta\right\} \sin \delta\right] \sin \lambda \\
& +\frac{U_{0}}{4 \pi}(1-\alpha) z\left[\left(\frac{\eta^{\prime}}{R^{3}}+B_{\eta^{\prime}}\right) \sin 2 \delta-\frac{2 q}{R^{3}} \cos 2 \delta\right] \cos \delta \cos \lambda,
\end{aligned}
$$

$$
\begin{aligned}
U_{z i \delta}{ }^{4}= & \frac{U_{0}}{4 \pi}(1-\alpha) z\left[\frac{1}{R\left(R+\eta^{\prime}\right)}-\xi^{\prime 2} A_{\eta^{\prime}}\right] \sin 2 \delta \sin \lambda \\
& +\frac{U_{0}}{4 \pi}(1-\alpha) z \xi^{\prime}\left[q A_{\eta^{\prime}}+\frac{1}{R^{3}} \sin 2 \delta-q A_{\eta^{\prime}} \cos 2 \delta\right] \cos \lambda \\
U_{z y}{ }^{4}= & -\frac{U_{0}}{4 \pi}(1-\alpha) z \xi^{\prime}\left[\frac{1}{R^{3}} \cos \delta+q A_{\eta^{\prime}} \sin \delta\right] \sin 2 \delta \sin \lambda \\
& +\frac{U_{0}}{4 \pi}(1-\alpha) z\left[\left(\frac{\eta^{\prime}}{R^{3}}-B_{\eta^{\prime}}\right)+\frac{2 q}{R^{3}} \sin 2 \delta+\left(\frac{\eta^{\prime}}{R^{3}}+B_{\eta^{\prime}}\right) \cos 2 \delta\right] \sin \delta \cos \lambda \\
U_{z z}{ }^{4}= & V_{z}^{4}+\frac{U_{0}}{4 \pi}(1-\alpha) z \xi^{\prime}\left[\frac{1}{R^{3}} \sin \delta-q A_{\eta^{\prime}} \cos \delta\right] \sin 2 \delta \sin \lambda \\
& +\frac{U_{0}}{4 \pi}(1-\alpha) z\left[-\left(\frac{\eta^{\prime}}{R^{3}}+B_{\eta^{\prime}}\right) \sin 2 \delta+\frac{2 q}{R^{3}} \cos 2 \delta\right] \sin \delta \cos \lambda
\end{aligned}
$$




$$
\left.\begin{array}{rl}
U_{x x}{ }^{5}= & \frac{U_{0}}{2 \pi} \alpha z \xi^{\prime} q\left[\frac{3 D_{0}}{R^{5}}+A_{\eta^{\prime}} \sin \delta\right] \sin \lambda \\
& +\frac{U_{0}}{2 \pi} \alpha z q\left[\frac{1}{R^{5}}\left(3 \xi^{\prime 2}-R^{2}\right) \sin \delta+Z_{0}\left(\xi^{\prime 2} C_{\eta^{\prime}}-A_{\eta^{\prime}}\right)\right] \cos \lambda, \\
U_{x y}{ }^{5}= & \frac{U_{0}}{2 \pi} \alpha z\left[K_{1}-\frac{1}{2} B_{\eta^{\prime}}+\frac{1}{2} B_{\eta^{\prime}} \cos 2 \delta\right] \sin \lambda \\
& +\frac{U_{0}}{2 \pi} \alpha z\left[K_{2}-\xi^{\prime} Z_{0} D_{\eta^{\prime}} \sin \delta+\frac{\xi^{\prime} q}{2} A_{\eta^{\prime}} \sin 2 \delta\right] \cos \lambda, \\
U_{x z}{ }^{5}= & V_{x}{ }^{5}+U_{z ; z^{\prime}}{ }^{5}, \\
U_{y x}{ }^{5}= & U_{x y^{5}}{ }^{5}, \\
U_{y y}{ }^{5}= & \frac{U_{0}}{2 \pi} \alpha z\left[q D_{0}\left(Y_{1}{ }^{2} C_{\xi^{\prime}}-A_{\xi^{\prime}}\right)+\left\{\left(\eta^{\prime} q \cos 2 \delta-2 Y_{1} D_{0}\right) A_{\xi^{\prime}}-\xi^{\prime} q A_{\eta^{\prime}} \sin { }^{2} \delta\right.\right. \\
& \left.\left.-B_{\xi^{\prime}} \sin 2 \delta\right\} \sin \delta\right] \sin \lambda+\frac{U_{0}}{2 \pi} \alpha z\left[-J_{1}+K_{5}\right. \\
& \left.-\frac{1}{2} q Z_{0}\left(2 A_{\eta^{\prime}}+D_{\eta^{\prime}}\right)(1-\cos 2 \delta)-B_{\eta^{\prime}} \sin 2 \delta \sin \delta\right] \cos \lambda, \\
U_{y z}{ }^{5}= & V_{y}{ }^{5}+\frac{U_{0}}{2 \pi} \alpha z\left[-q Y_{1} D_{0} D C_{\xi^{\prime}}+\left(D \sin \delta-Y_{1} \cos \delta\right) D_{0} A_{\xi^{\prime}}\right. \\
& \left.-\left\{B_{\xi^{\prime}} \cos 2 \delta+q\left(\eta^{\prime} A_{\xi^{\prime}}+\frac{1}{2} \xi^{\prime} A_{\eta^{\prime}}\right) \sin 2 \delta\right\} \sin \delta\right] \sin \lambda \\
& -\frac{U_{0}}{2 \pi} \alpha z\left[J_{2}+K_{0}+\frac{1}{2} q Z_{0}\left(2 A_{\eta^{\prime}}+D_{\eta^{\prime}}\right) \sin 2 \delta+B_{\eta^{\prime}} \cos 2 \delta \sin \delta\right] \cos \lambda,
\end{array}\right\}
$$

$$
\begin{aligned}
U_{z x}{ }^{5}= & -\frac{U_{0}}{2 \pi} \alpha z\left[K_{3}+\frac{1}{2} B_{\eta^{\prime}} \sin 2 \delta\right] \sin \lambda \\
& +\frac{U_{0}}{2 \pi} \alpha z\left[K_{4}-\frac{1}{2} \xi^{\prime} q A_{\eta^{\prime}}-\xi^{\prime} Z_{0} D_{\eta^{\prime}} \cos \delta+\frac{1}{2} \xi^{\prime} q A_{\eta^{\prime}} \cos 2 \delta\right] \cos \lambda, \\
U_{z y^{5}}{ }^{5} & U_{y z^{5}}, \\
U_{z z}{ }^{5}= & V_{z}^{5}+\frac{U_{0}}{2 \pi} \alpha z\left[q D_{0}\left(C_{\xi^{\prime}} D^{2}-A_{\xi^{\prime}}\right)+\left(-\eta^{\prime} q A_{\xi^{\prime}} \cos 2 \delta+B_{\xi^{\prime}} \sin 2 \delta\right.\right. \\
& \left.\left.-\xi^{\prime} q A_{\eta^{\prime}} \cos ^{2} \delta\right) \sin \delta+2 D_{0} D A_{\xi^{\prime}} \cos \delta\right] \sin \lambda+\frac{U_{0}}{2 \pi} \alpha z\left[-J_{3}+K_{7}\right. \\
& \left.-\frac{1}{2} q Z_{0}\left(2 A_{\eta^{\prime}}+D_{\eta^{\prime}}\right)(1+\cos 2 \delta)+B_{\eta^{\prime}} \sin 2 \delta \sin \delta\right] \cos \lambda,
\end{aligned}
$$




$$
\begin{aligned}
& X^{2}=\xi^{\prime 2}+q^{2}, \quad Y^{2}=\eta^{\prime 2}+q^{2}, \quad Y_{1}=\eta^{\prime} \cos \delta+q \sin \delta, \\
& \left.D=\eta^{\prime} \sin \delta-q \cos \delta, \quad D_{0}=D+z, \quad Z_{0}=z-q \cos \delta,\right\} \\
& A_{\xi^{\prime}}=\frac{2 R+\xi^{\prime}}{R^{3}\left(R+\xi^{\prime}\right)^{2}}, \quad A_{\eta^{\prime}}=\frac{2 R+\eta^{\prime}}{R^{3}\left(R+\eta^{\prime}\right)^{2}}, \\
& B_{\xi^{\prime}}=\frac{1}{R\left(R+\xi^{\prime}\right)}-q^{2} A_{\xi^{\prime}}, \quad B_{\eta^{\prime}}=\frac{1}{R\left(R+\eta^{\prime}\right)}-q^{2} A_{\eta^{\prime}}, \\
& \begin{array}{l}
C_{\xi^{\prime}}=\frac{8 R^{2}+9 R \xi^{\prime}+3 \xi^{\prime 2}}{R^{5}\left(R+\xi^{\prime}\right)^{3}}, \quad C_{\eta^{\prime}}=\frac{8 R^{2}+9 R \eta^{\prime}+3 \eta^{\prime 2}}{R^{5}\left(R+\eta^{\prime}\right)^{3}}, \\
D_{\eta^{\prime}}=A_{\eta^{\prime}}-q^{2} C_{\eta^{\prime}},
\end{array} \\
& P_{x x}=\frac{\xi^{\prime} q}{R^{3}} \sin \lambda+\xi^{\prime 2} q A_{\gamma^{\prime}} \cos \lambda, \\
& \left.\begin{array}{l}
P_{x y}=\frac{1}{R^{3}}\left[\xi^{\prime 2} \sin \delta+\eta^{\prime} D\right] \sin \lambda-\xi^{\prime 3}\left[\frac{D}{Y^{2} R^{3}}-A_{\eta^{\prime}} \sin \delta\right] \cos \lambda, \\
P_{x z}=\frac{1}{R^{3}}\left[\xi^{\prime 2} \cos \delta+\eta^{\prime} Y_{1}\right] \sin \lambda-\xi^{\prime 3}\left[\frac{Y_{1}}{Y^{2} R^{3}}-A_{\eta^{\prime}} \cos \delta\right] \cos \lambda,
\end{array}\right\} \\
& P_{y x}=\frac{q}{R^{3}}\left[q \sin \delta-\frac{\eta^{\prime 3}}{X^{2}} \cos \delta\right] \sin \lambda+\xi^{\prime} q\left[q A_{y^{\prime}} \sin \delta+\frac{1}{R^{3}} \cos \delta\right] \cos \lambda, \\
& P_{y y}=\left[\frac{\xi^{\prime} q}{2 R^{3}}+\left\{\frac{\xi^{\prime}}{2 R\left(R+\eta^{\prime}\right)}+\eta^{\prime} B_{\xi^{\prime}}\right\} \sin 2 \delta-q\left\{\begin{array}{c}
1 \\
R\left(R+\xi^{\prime}\right)
\end{array}\right.\right. \\
& \left.\left.+\frac{1}{2}\left(\eta^{\prime 2}-q^{2}\right) A_{\xi^{\prime}}\right\} \cos 2 \delta\right] \sin \lambda+\left[\frac{1}{2} \xi^{\prime 2} q A_{\eta^{\prime}}+\frac{\xi^{\prime 2}+\eta^{\prime 2}-q^{2}}{2 R^{3}} \sin 2 \delta\right. \\
& \left.-q\left\{\frac{\eta^{\prime}}{2 R^{3}}+\frac{1}{R\left(R+\eta^{\prime}\right)}-\frac{1}{2} q^{2} A_{\eta^{\prime}}\right\} \cos 2 \delta\right] \cos \lambda, \\
& P_{y z}=\left[\left\{\frac{\eta^{\prime}}{R\left(R+\xi^{\prime}\right)}+\frac{\xi^{\prime}}{2 R\left(R+\eta^{\prime}\right)}\right\}+q\left\{\frac{1}{R\left(R+\xi^{\prime}\right)}+{ }^{1}\left(\eta^{\prime 2}-q^{2}\right) A_{\xi^{\prime}}\right\} \sin 2 \delta\right. \\
& \left.+\left\{\frac{\xi^{\prime}}{2 R\left(R+\eta^{\prime}\right)}+\eta^{\prime} B_{\xi^{\prime}}\right\} \cos 2 \delta\right] \sin \lambda+\left[\begin{array}{c}
1 \\
2 R
\end{array}+q\left\{\begin{array}{c}
\eta^{\prime} \\
2 R^{3}
\end{array}+\begin{array}{c}
1 \\
R\left(R+\eta^{\prime}\right)
\end{array}\right.\right. \\
& \left.\left.-\frac{1}{2} q^{2} A_{\eta^{\prime}}\right\} \sin 2 \delta+\frac{\xi^{\prime 2}+\eta^{\prime 2}-q^{2}}{2 R^{3}} \cos 2 \delta\right] \cos \lambda \text {, } \\
& P_{z x x}=\frac{q}{R^{3}}\left[q \cos \delta+\frac{\eta^{\prime 3}}{X^{2}} \sin \delta\right] \sin \lambda+\xi^{\prime} q\left[q A_{\eta^{\prime}} \cos \delta-\frac{1}{R^{3}} \sin \delta\right] \cos \lambda, \\
& P_{z y}=\left[\left\{\frac{\eta^{\prime}}{R\left(R+\xi^{\prime}\right)}+\frac{\xi^{\prime}}{2 R\left(R+\eta^{\prime}\right)}\right\}-q\left(\frac{\xi^{\prime}}{2 R^{3}}+\eta^{\prime 2} A_{\xi^{\prime}}\right) \sin 2 \delta\right. \\
& \left.-\frac{1}{2}\left\{\frac{\xi^{\prime} \eta^{\prime}}{R^{3}}+\frac{\xi^{\prime}}{R\left(R+\eta^{\prime}\right)}+\eta^{\prime}\left(\eta^{\prime 2}-q^{2}\right) A_{\xi^{\prime}}\right\} \cos 2 \delta\right] \sin \lambda
\end{aligned}
$$




$$
\begin{aligned}
& +\left[\begin{array}{c}
1 \\
2 R
\end{array}-q\left(\begin{array}{l}
\eta^{\prime} \\
R^{3}
\end{array}+\frac{1}{2} \xi^{\prime 2} A_{\eta^{\prime}}\right) \sin 2 \delta-\frac{\xi^{\prime 2}+\eta^{\prime 2}-q^{2} \cos 2 \delta}{2 R^{3}}\right] \cos \lambda, \\
P_{z z}= & {\left[\left\{\begin{array}{c}
\xi^{\prime} \\
2 R\left(R+\eta^{\prime}\right)
\end{array}+\eta^{\prime} B_{\xi^{\prime}}\right\} \sin 2 \delta-q\left\{\begin{array}{c}
1 \\
R\left(R+\xi^{\prime}\right)
\end{array}+\frac{1}{2}\left(\gamma^{\prime 2}-q^{2}\right) A_{\xi^{\prime}}\right\} \cos 2 \delta\right] \sin \lambda } \\
& +\left[\begin{array}{c}
\xi^{\prime 2}+\gamma^{\prime 2}-q^{2} \\
2 R^{3}
\end{array} \sin 2 \delta-q\left\{\begin{array}{c}
\eta^{\prime} \\
\left.\left.2 R^{3}+\frac{1}{R\left(R+\eta^{\prime}\right)}-\frac{q^{2}}{2} A_{\gamma^{\prime}}\right\} \cos 2 \delta\right] \cos \lambda,
\end{array}\right\}\right.
\end{aligned}
$$

$$
\begin{aligned}
& V_{x}{ }^{4}=\frac{U_{01}}{2 \pi}(1-\alpha)\left[\begin{array}{l}
1 \\
R
\end{array} \cos \delta-\frac{q}{R\left(R+\gamma^{\prime}\right)} \sin \delta\right] \sin \lambda \\
& +{ }_{2 \pi}^{U_{\prime \prime}}(1-\alpha) \underset{R\left(R+\gamma^{\prime}\right)}{\xi^{\prime}} \cos \delta \cos \lambda, \\
& V_{y}{ }^{4}=\frac{U_{0}^{\prime \prime}}{2 \pi}(1-\alpha)\left[\begin{array}{c}
\eta^{\prime} \\
R\left(R+\xi^{\prime}\right)
\end{array}+\frac{\xi^{\prime}}{2 R\left(R+\eta^{\prime}\right)}(1-\cos 2 \delta)\right] \sin \lambda \\
& +\frac{U_{0}}{4 \pi}(1-\alpha)\left[\frac{1}{R}(1+\cos 2 \delta)+\frac{q}{R\left(R+\eta^{\prime}\right)} \sin 2 \delta\right] \cos \lambda, \\
& -\frac{U_{0}}{4 \pi}\left(1-\alpha^{\prime}\right)\left[\begin{array}{c}
q \\
R\left(R+\gamma_{i}^{\prime}\right)
\end{array}(1-\cos 2 \delta)+\frac{1}{R} \sin 2 \delta\right] \cos \lambda,
\end{aligned}
$$

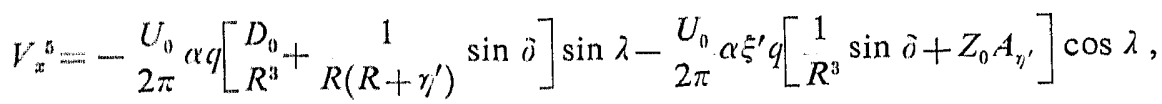

$$
\begin{aligned}
& V_{y}^{3}=\frac{U_{0}}{2 \pi} \alpha\left[-q Y_{1} D_{11} A_{\xi^{\prime}}+\frac{1}{R\left(R+\xi^{\prime}\right)}\left(D+D_{0}\right) \sin \delta+\frac{\xi^{\prime}}{R\left(R+\gamma^{\prime}\right)} \sin ^{2} \delta\right] \sin \lambda
\end{aligned}
$$$$
+{ }_{2 \pi}^{U_{0}} \alpha\left[Z_{01} B_{\eta^{\prime}} \sin \delta-\frac{q}{R^{3}} D_{11} \cos \delta-\frac{q}{2 R\left(R+\eta^{\prime}\right)} \sin 2 \delta\right.
$$

$$
\begin{aligned}
& \left.++^{\xi^{\prime 2}+\gamma^{\prime 2}} \operatorname{Rin}^{3} \delta\right] \cos \lambda \\
& V_{z}^{s}=\frac{U_{0}}{2 \pi} \alpha\left[q D D_{0} A_{\xi^{\prime}}+\frac{1}{R\left(R+\xi^{\prime}\right)}\left(Y_{1} \sin \delta+D_{0} \cos \delta\right)+\frac{\xi^{\prime}}{2 R\left(R+\eta^{\prime}\right)} \sin 2 \delta\right] \sin \lambda \\
& +\frac{U_{0}}{2 \pi} \alpha\left[\begin{array}{c}
q D_{0} \\
R^{3}
\end{array} \sin \delta+Z_{0} B_{r}, \cos \delta+{ }^{\xi^{\prime 2}+\gamma^{\prime 2}} \underset{2 R^{3}}{2} \sin 2 \delta\right. \\
& \left.+\frac{q}{R\left(R+\eta^{\prime}\right)} \sin ^{2} \delta\right] \cos \lambda \text {, }
\end{aligned}
$$

$$
I_{1}=\int_{\partial x}^{\partial F_{1}} d y^{\prime}=\begin{array}{cc}
1-\alpha & \xi^{\prime} \\
\alpha & X^{2} R(R+D)^{2}
\end{array}\left[\eta^{\prime} Y^{2}+R\left(\gamma^{\prime} D-q Y_{1}\right)\right],
$$




$$
\left.\begin{array}{rl}
I_{2}= & \int \frac{\partial F_{1}^{\prime}}{\partial y} d \eta^{\prime}=-\frac{1-\alpha}{\alpha} \frac{1}{2 X^{2} R(R+D)^{2}}\left[\xi^{\prime 2}\left\{2 \eta^{\prime} Y_{1}-q(R-D)\right\}\right. \\
& \left.+q(R+D)\left(R^{2}+Y^{2}\right)\right], \\
I_{3}= & \int \frac{\partial F_{3}}{\partial x} d \eta^{\prime}=-\frac{1-\alpha}{\alpha} X^{2} R(R+D) \\
I_{4}= & \int \frac{\partial F_{3}}{\partial y} d \eta^{\prime}=-\frac{1-\alpha}{\alpha}{\xi^{\prime}}^{\prime} R(R+D) \\
J_{1}= & \frac{1}{R^{3}}\left[q \sin \delta(1-2 \cos 2 \delta)+\eta_{0} \sin 2 \delta\right], \\
J_{2}= & \frac{1}{R^{3}}\left[D_{0} \cos 2 \delta+2 q \sin 2 \delta \sin \delta\right], \\
J_{3}= & \frac{1}{R^{3}}\left[q \sin \delta-D_{0} \sin 2 \delta+2 q \cos 2 \delta \sin \delta\right], \\
K_{1}= & \frac{1}{2 R^{5}}\left[6 q Y_{1} D_{0}-2 R^{2} D_{0} \sin \delta+R^{2} q \sin 2 \delta\right], \\
K_{2}= & \frac{\xi}{2 R^{5}}\left[\left(3 q^{2}-R^{2}\right)+6 q D_{0} \cos \delta-\left(3 q^{2}-R^{2}\right) \cos 2 \delta\right], \\
K_{3}= & \frac{1}{2 R^{5}}\left[q\left(R^{2}+6 D D_{0}\right)+2 R^{2} D_{0} \cos \delta-R^{2} q \cos 2 \delta\right], \\
K_{4}= & \frac{\xi}{2 R^{5}}\left[-6 q D_{0} \sin \delta+\left(3 q^{2}-R^{2}\right) \sin 2 \delta\right], \\
K_{5}= & \frac{3 q}{2 R^{5}}\left[Z_{0} \eta^{\prime}+2 Y_{1}^{2} \sin \delta+Z_{0}\left(\eta^{\prime} \cos 2 \delta+2 q \sin 2 \delta\right)\right], \\
K_{6}= & \frac{3 q}{2 R^{5}}\left[q D_{0}+\left(2 D_{0} D-q^{2}\right) \cos \delta-q D_{0} \cos 2 \delta+q^{2} \cos 2 \delta \cos \delta\right], \\
K_{7}= & \frac{3 q}{2 R^{5}}\left[Z_{0} \eta^{\prime}+2 D^{2} \sin \delta-Z_{0}\left(\eta^{\prime} \cos 2 \delta+2 q \sin 2 \delta\right)\right] .
\end{array}\right\}
$$

\section{Program System}

One of our main purposes is to establish the program system for the static deformation due to the fault model described above. The computer program developed enables us to draw contour maps of displacement or strain fields on an arbitrary plane in the medium. The structure of the program system is shown in the flow chart (Fig. 3).

\subsection{Input parameters}

(a) $V P, V S, U N, N F$

$V P$ and $V S$ are the $P$ and $S$ wave velocity of the medium in $\mathrm{km} / \mathrm{sec}$. $U N$ 
Table 1. The roles of important input parameters.

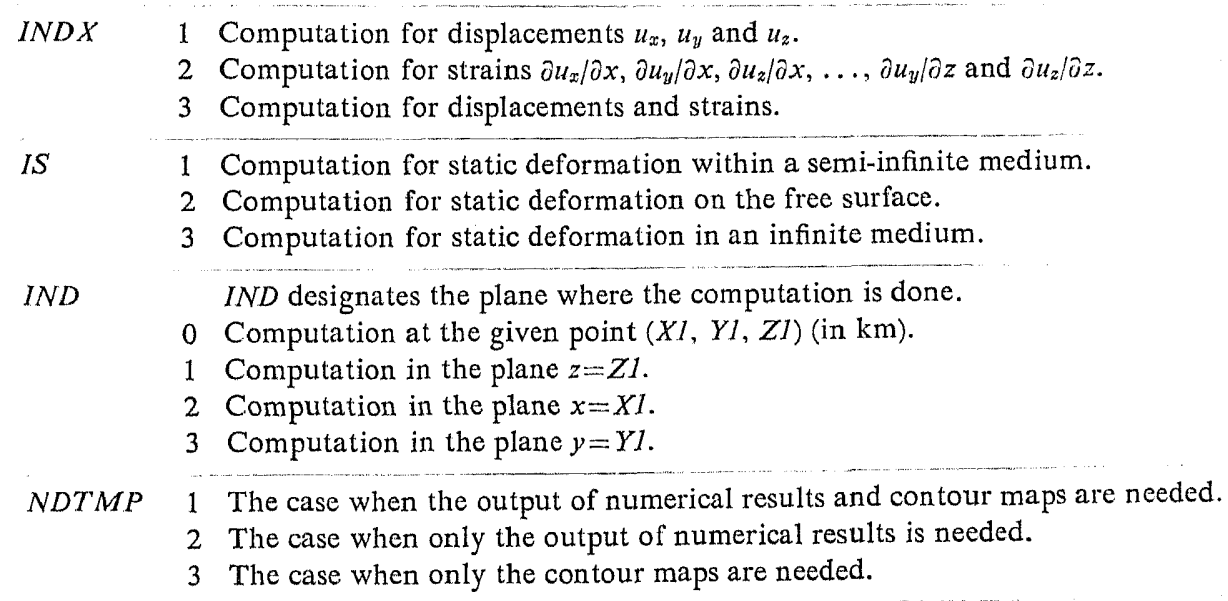

is the unit length in $\mathrm{km}$, which is the characteristic length used throughout the computation and referred to as $H$ in the text. NF is the number of the faults that is necessary to input when calculating the deformation fields due to multiple faults with different orientations.

(b) INDX,IS, IND, NDTMP, XI,Y1,Z1

The roles of these parameters are explained in Table 1 . When IND is not zero, the region of the computation is a square with a side $4 H$, whose center is placed at $(X 1, Y 1, Z 1)$ in $\mathrm{km}$.

(c) Fault parameters

$D S M(\mathrm{~m}), A W(\mathrm{~km}), S L I P, D I P$ (degree), and $D P T(\mathrm{~km})$ correspond to $U_{0}, w, \lambda, \delta$ and $d_{0}$ respectively. $x$ and $y$ coordinates of the two lower corners of the fault plane are given by $(X O, Y O)$ and $(X 2, Y 2)$ in $\mathrm{km}$. In such a case as in Fig. $1, Y O=Y 2=0$ and $X O=-X 2=L$.

(d) $C R(i)(i=1,11)$

$C R(i)$ is contour value and the inequality $C R(i)<C R(i+1)$ must be kept.

\subsection{Contour map}

To draw the contour map, computation is carried out at $41 \times 41(=1681)$ mesh points in a square region with a side $4 H$ designated by $X 1, Y 1, Z 1$, and IND (Fig. 4(a)). Inside a small square with a side $0.1 \mathrm{H}$, deformations are obtained from values at four corner points of the square by the interpolation technique. If the fault lies in this square, however, the above interpolation is not appropriate for drawing an accurate contour map because of the abrupt change of the deformation field. In such a case, in the present program, the computation is done at all the points $(6 \times 4$ points) in the small squares in the vicinity of the fault. 


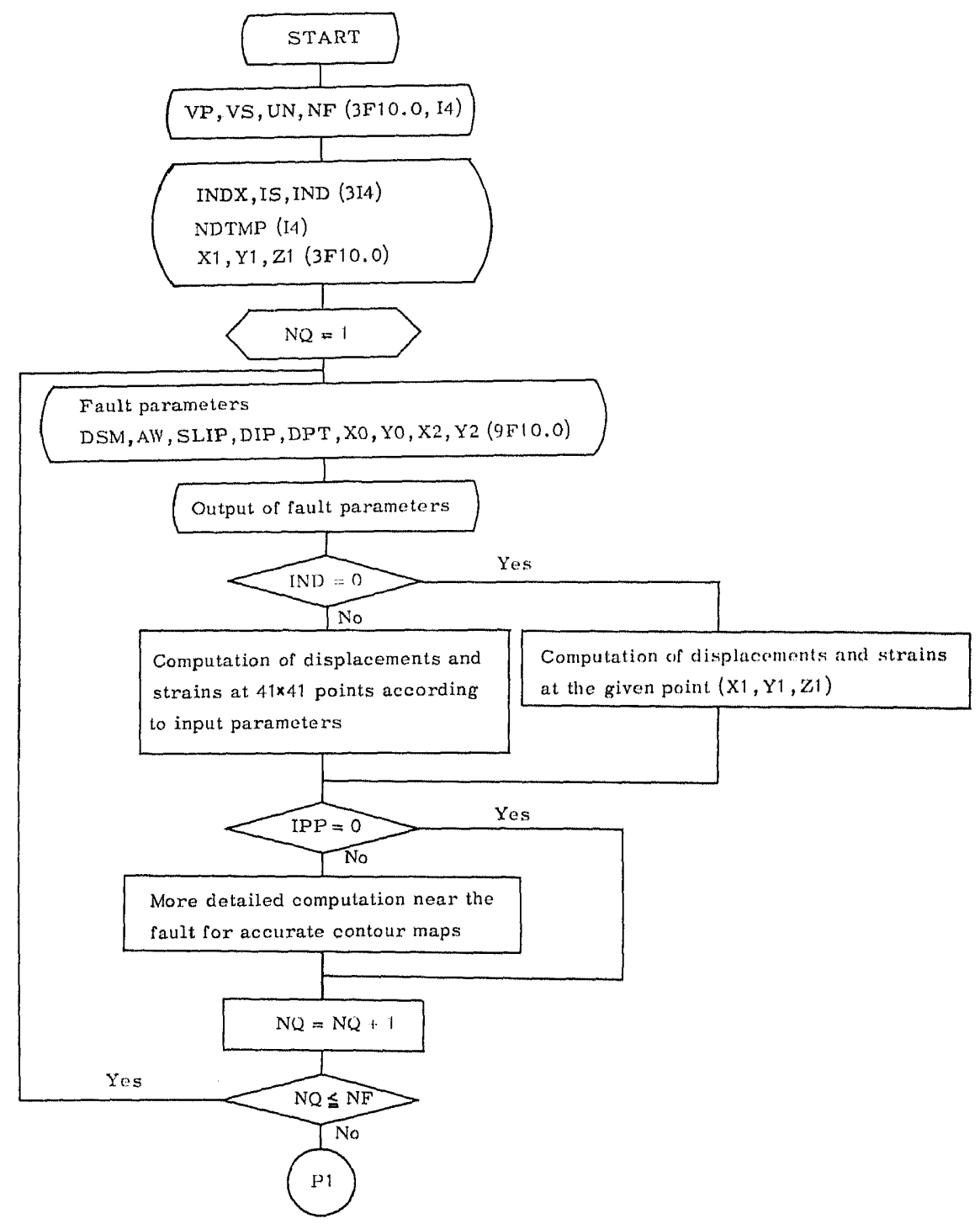

Fig. 3. Flow chart. IPP is set to 1 when the fault intersects the plane where the contour map is draw and otherwise is 0 .

As an example, a vertical cross-section near the fault is shown in Fig. 4(b), when $d_{0} / H=1.0, w / H=1.0=w^{\prime}$ and $\delta=60^{\circ}$ taking $X I=0$. If $I N D=2$ is designated, deformation fields are computed for this cross-section. Since the fault crosses the plane, to draw the map, a detailed computation is needed near the fault without the interpolation. 


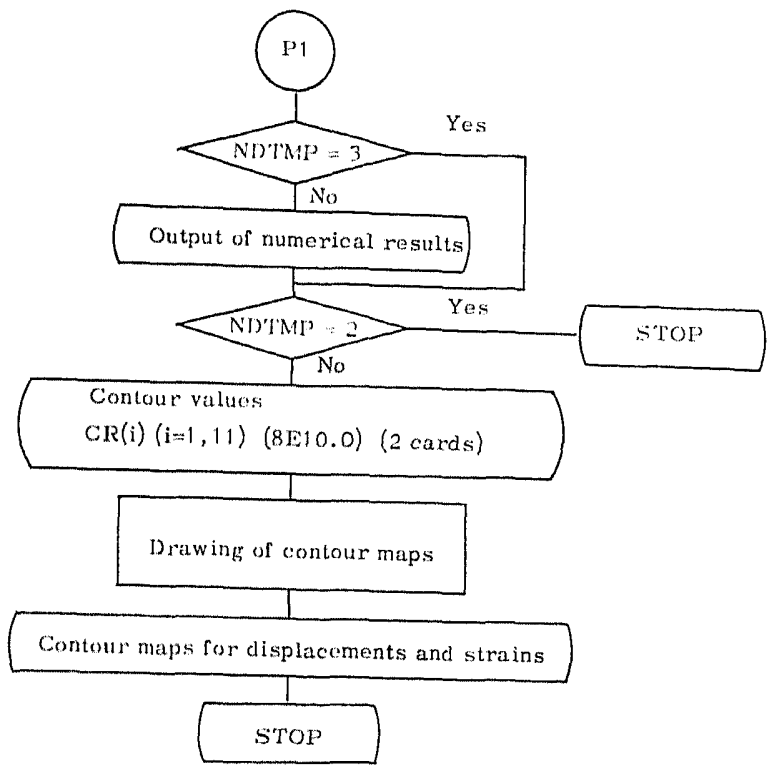

Fig. 3 (continued)

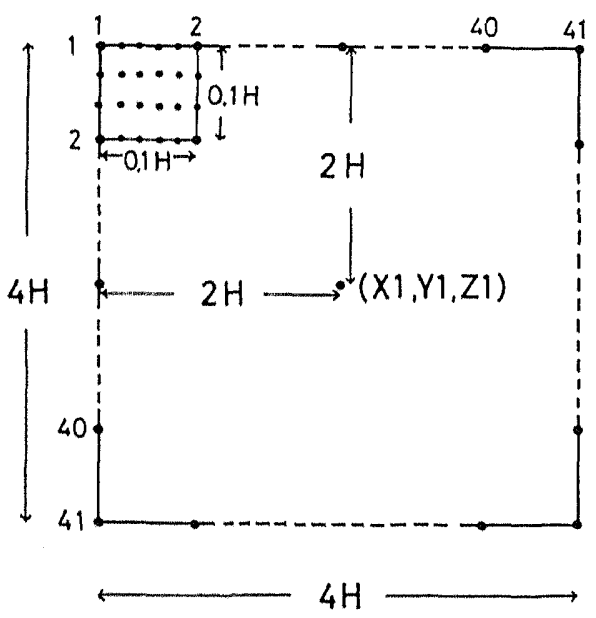

(a)

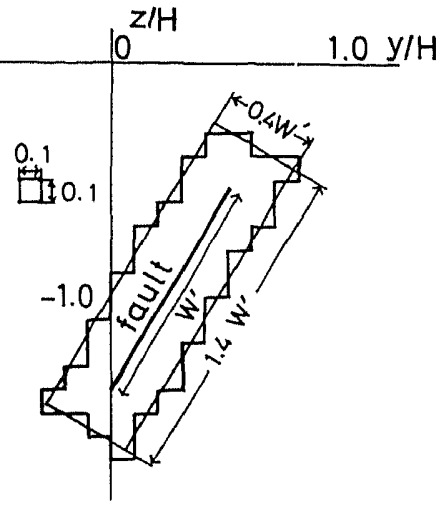

(b)

Fig. 4. (a) Illustration of square plane where contour map is drawn. This square consists of $41 \times 41$ mesh points where static deformations are computed. At $6 \times 4$ points inside a small square with a side of $0.1 \mathrm{H}$, deformations are obtained by using the four point formula of bivariate interpolation. (b) A vertical cross section $(x / H=0)$ in the vicinity of the fault (expressed by a solid line of length $\left.w^{\prime}\right)$. To obtain an accurate contour map near the fault, detailed computations are necessary in the region surrounded by the heavy solid line whose area is about $1.4 w^{\prime} \times 0.4 w^{\prime}$. It is found by trial and error that this area is sufficient to obtain accurate mapping. 


\section{Numerical Examples}

By the use of the computer program described in the previous section, several numerical examples are presented, for all of which, $V_{\mathrm{P}}=6.1 \mathrm{~km} / \mathrm{sec}$, $V_{\mathrm{S}}=3.5 \mathrm{~km} / \mathrm{sec}, 2 L / H=2, w / H=1$ and $U_{0} / H=1 \times 10^{-4}$ are assumed. The dipangle and fault depth is set to $\delta=60^{\circ}$ and $d_{0} / H=1.4$ unless stated otherwise. The unit of $U_{0} \times 10^{-2}$ for displacements and $\left(U_{0} / H\right) \times 10^{3}$ for strains are chosen.

\subsection{Strain fields in the vertical cross section}

First, the strain field is compared with the displacement field. In Figs. $5(\mathrm{a})$ and $5(\mathrm{~b})$, the vertical displacement $u_{z}$ and the strain component $\partial u_{z} / \partial y$ in the $y z$ plane are shown for a pure dip-slip fault $\left(\lambda=90^{\circ}\right)$. It is seen that the pattern of the strain field can roughly be found from that of the corresponding displacement field. For example, two curves $\left(P_{1} P_{2} P_{3}\right.$ and $\left.Q_{1} Q_{2} Q_{3}\right)$ in Fig. $5(\mathrm{a})$, which connect peaks and troughs of $u_{z}$ at various depths, coincide with two zero lines of $\partial u_{z} / \partial y$ below the lower edge of the fault in Fig. 5(b). On the other hand, for a region where $\left|\partial u_{z} / \partial y\right|$ is large, $u_{z}$ shows a sharp change. Such a comparison can be used as one of the qualitative checks for our analytical expressions and for the program system.

Next, effect of the free surface on the strain field is investigated. This effect can be clarified by comparing the strain patterns for a semi-infinite medium (Model I) with those for an infinite medium (Model II). Figure 5(c) shows the pattern of $\partial u_{z} / \partial y$ for Model II, with the same fault parameters and velocity structure as those in Fig. 5(b). Comparing Fig. 5(b) with Fig. 5(c), two remarkable differences are found. First, only in Model I, a saddle point appears at about $z=-H$ in the lower-block side of the fault, which is indicated by a mark in Fig. 5(b). Secondly, a concentration of contour lines is found in Model I above the upper edge of the fault plane, which implies that peaks of $u_{z}$ along a line $z=$ const. in the $y z$ plane are sharper for Model $I$ than for Model II, as illustrated in Fig. 6. As one more characteristic of the effect of the free surface, the "reverse area" appears in Model I, where the sense of the deformation is opposite to that of Model II. For this example, the strain component $\partial u_{y} / \partial z$ for a pure dip-slip fault is exhibited in Fig. 7. For Mode1 I, it has negative values in the area $(y / H \geq 1.4)$ near the free surface, while, for Model II, positive values with a simpler pattern in the corresponding area. This effect is remarkable in the strain components $\partial u_{*} / \hat{\partial} z$ and $\partial u_{y} / \partial z$ (since $\partial u_{x} / \partial z$ vanishes in the $y z$ plane for a pure dip-slip fault, its pattern is investigated in the plane of $x / H=1$ ).

It is clear from Figs. 5 and 7 that the above effect does not extend below the center of the fault plane, where the contribution from $\boldsymbol{u}^{1}$ in Eq. (2.1) is predominant. This is generally seen in other components for a pure dip-slip fault. 


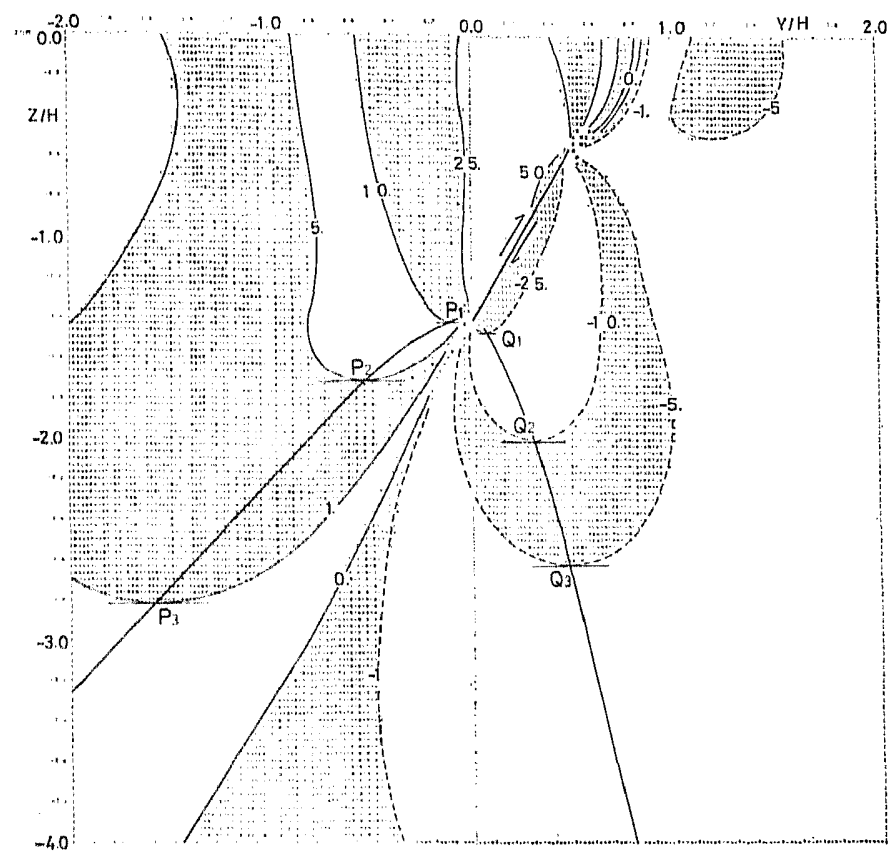

Fig. 5(a)

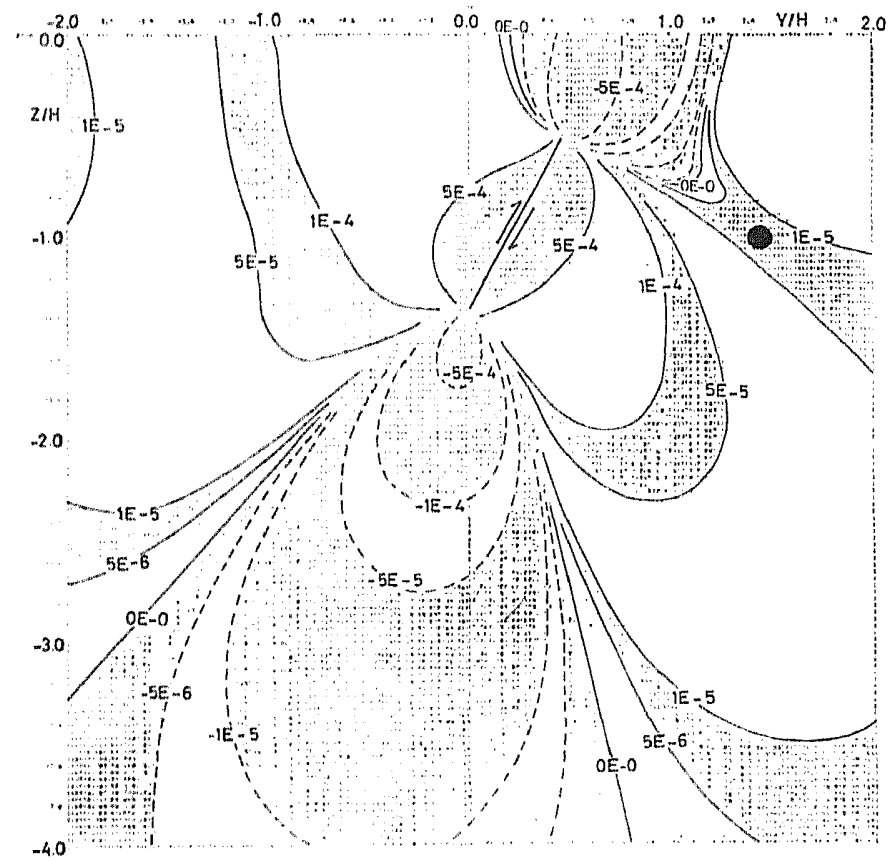

Fig. 5(b) 


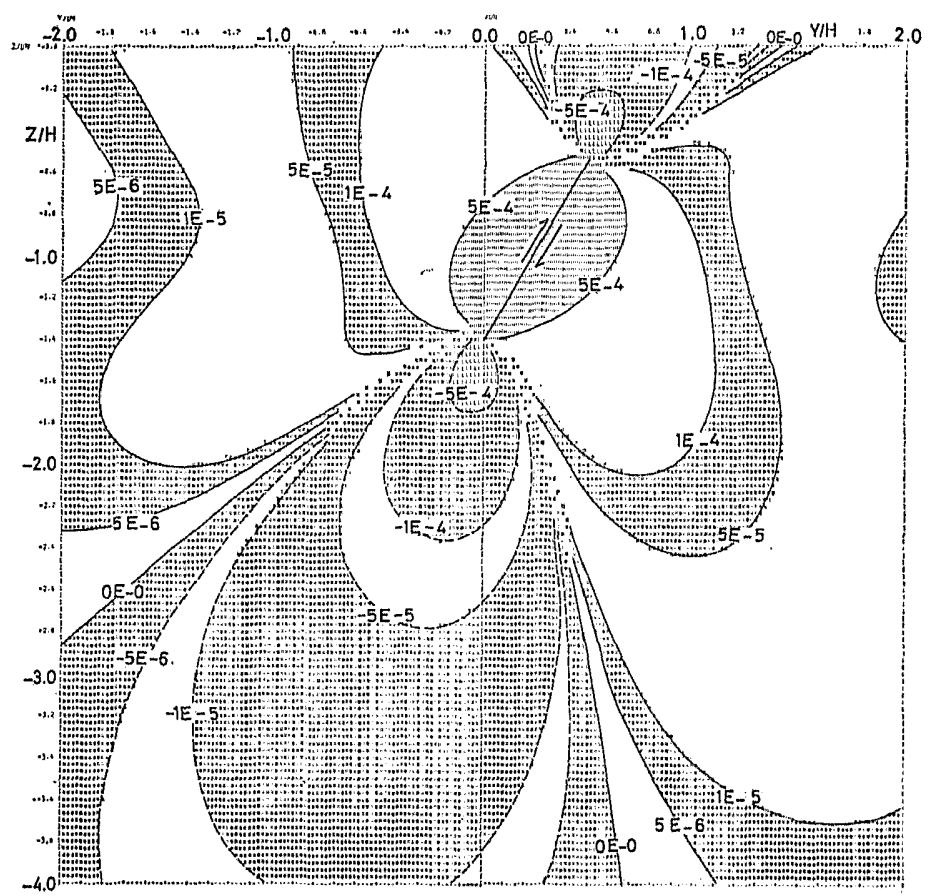

Fig. 5(c)

Fig. 5. Vertical displacement $u_{z}$ with unit of $U_{0} \times 10^{-2}$ and $\partial u_{z} / \partial y$ with $\left(U_{0} / H\right) \times 10^{3}$ in the $y z$ plane for a pure dip-slip fault. In all the following figures for strains, contour values are expressed by special symbols, for example 5E- -5 , which means $5 \times 10^{-5}$. Model I and II are the cases of a semi-infinite medium and an infinite medium respectively. Two curves of $\overline{\mathrm{P}}_{1} \mathrm{P}_{2} \mathrm{P}_{3}$ and $\mathrm{Q}_{1} \mathrm{Q}_{2} \mathrm{Q}_{3}$ in (a) correspond to zero lines in (b). Comparing (b) with (c), a saddle point (indicated by a mark $\bullet$ ) and concentration of contour lines are found only in (b). (a) $u_{x}$, (b) $\partial u_{z} / \hat{\hat{y} y}$ for Model I, (c) $\partial u_{z} / \partial y$ for Model II.

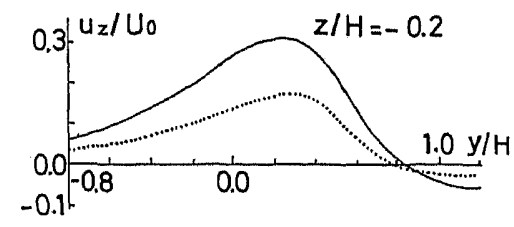

Fig, 6. Vertical displacement $u_{z}$ with $y$ at depth of $z / H=-0.2$ in the $y z$ plane due to the pure dipslip fault in Fig. 5. Solid and dotted curves are the cases of Model I and II respectively.

The above characteristic effect is also found for a pure strike-slip fault $\left(\lambda=0^{\circ}\right)$. Patterns of the strain component $\partial u_{y} / \partial z$ in the plane $(x / H=1)$ are presented in Fig. 8. In this case, two saddle points are found for Model I. The reverse area spreads from the free surface to the depth of about $0.6 \mathrm{H}$ in the upper-block side of the fault. The concentration of contour lines is again found on the boundary of this reverse area.

Thus it can be concluded that existence of the free surface introduces 


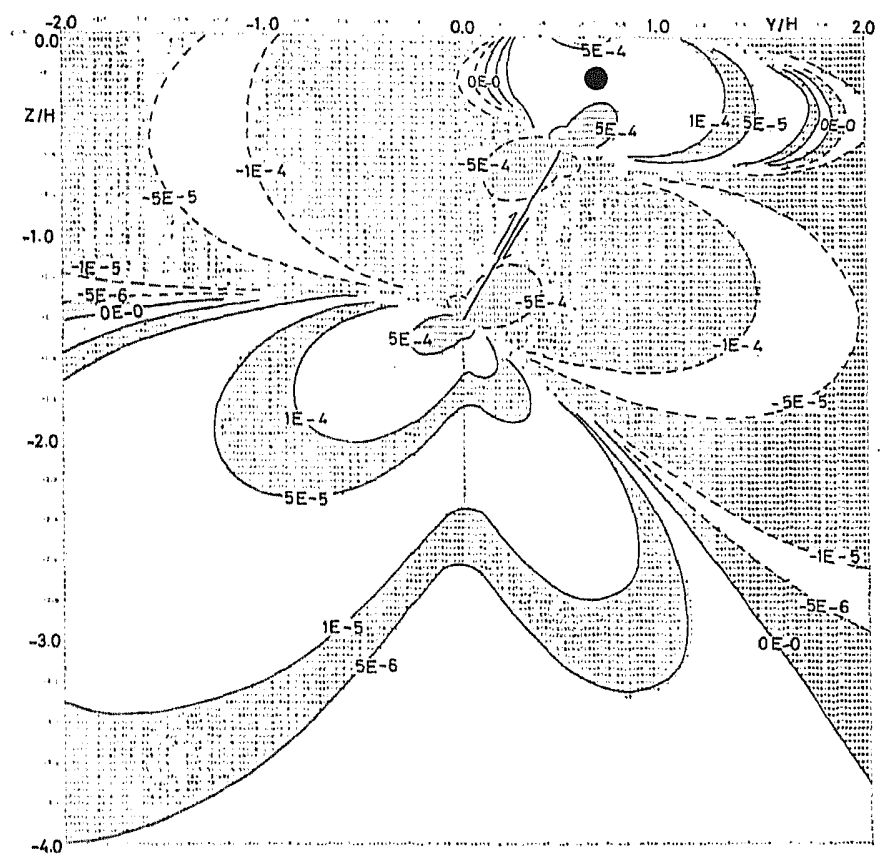

(a)

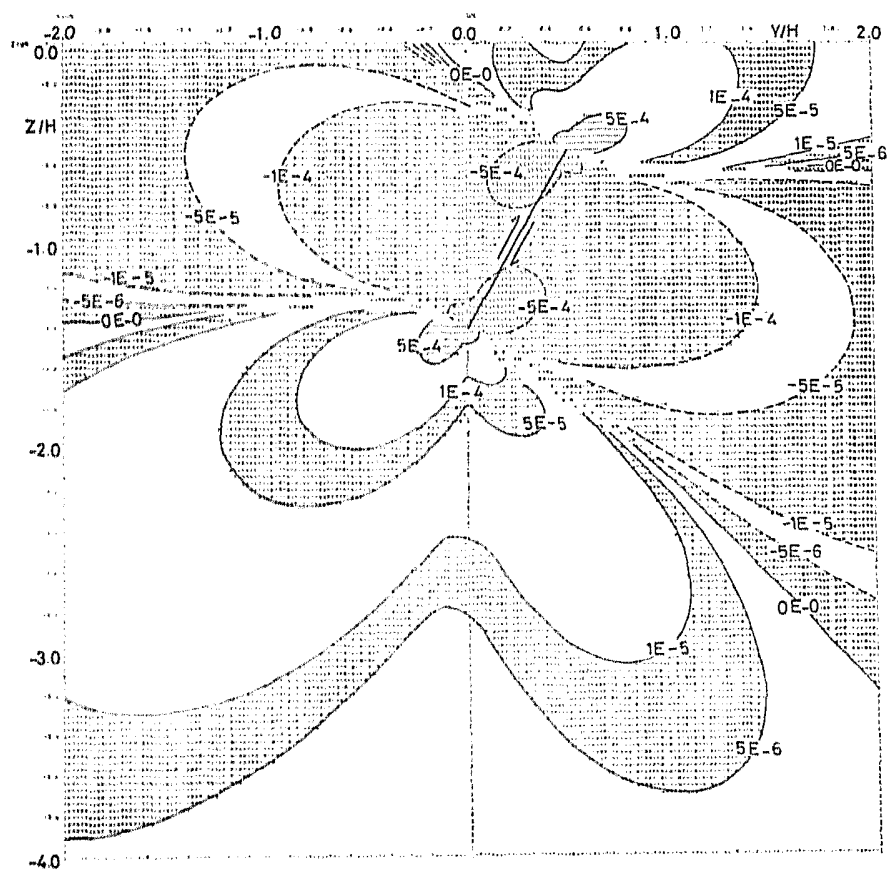

(b)

Fig. 7. $\partial u_{y} / \partial z$ in the $y z$ plane due to the pure dip-slip fault. The "reverse area" is found in (a) in the region of $y / H \gtrsim 1.4$ near the free surface. (a) Model $I$, (b) Model II. 


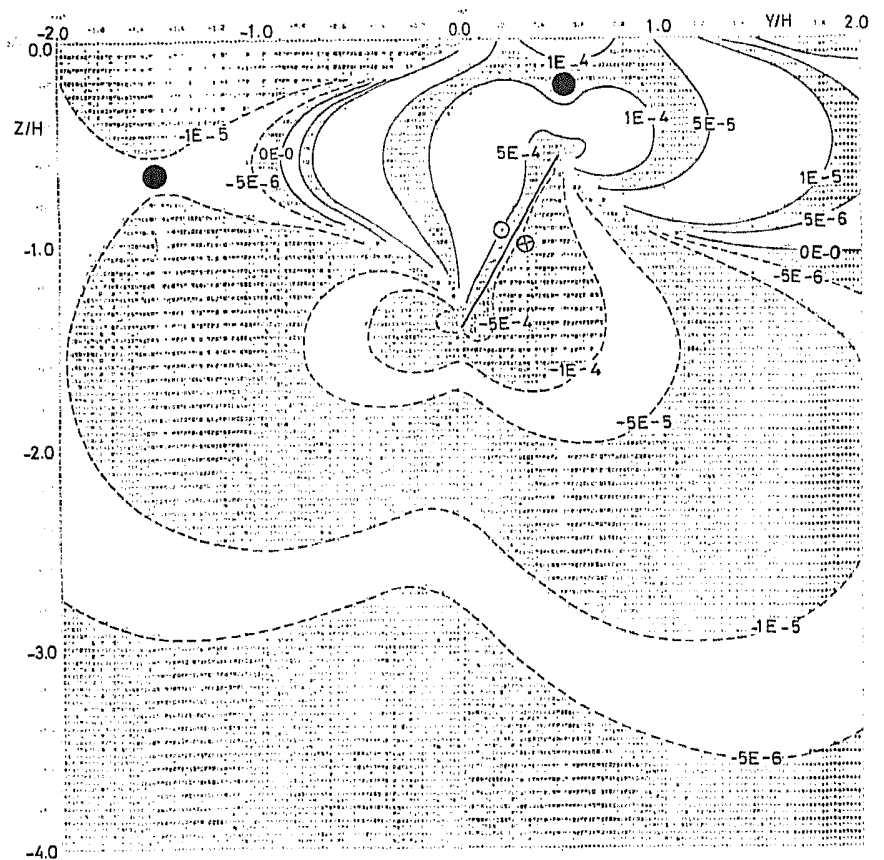

(a)

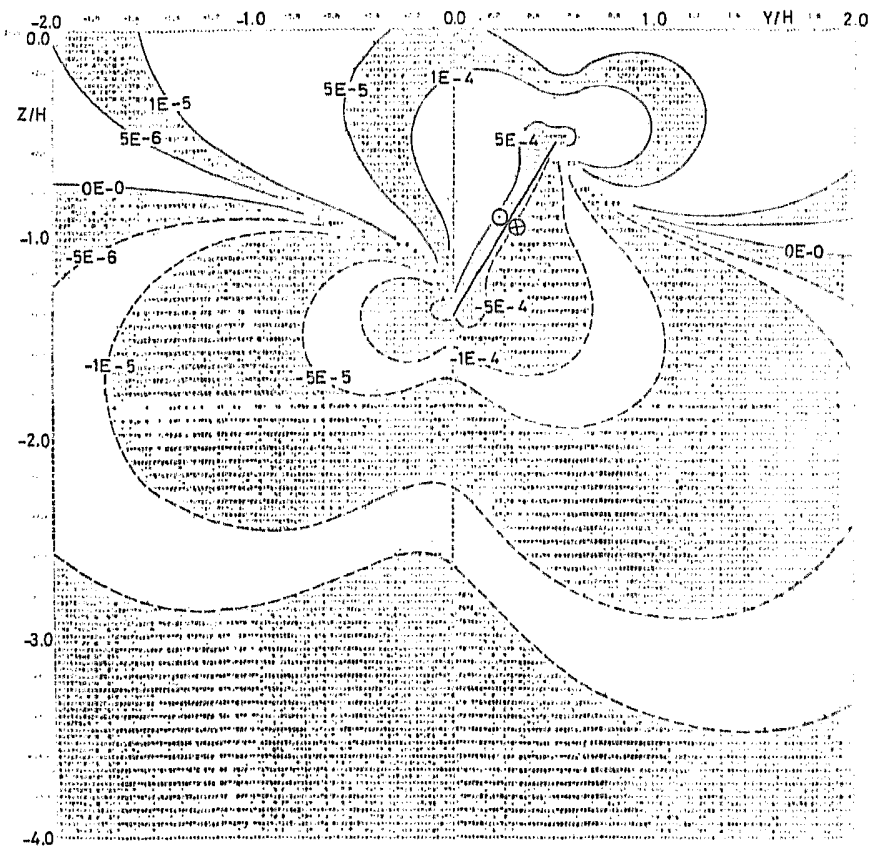

(b)

Fig. 8. $\partial u_{y} / \partial z$ in the vertical plane of $x / H=1$ due to a pure strike-slip fault. Effect of the free surface appears clearly in the pattern for Model I. (a) Model I, (b) Model Ir. 
saddle points and concentration of contour lines and also the reverse area, in the vertical cross section, for the dip- and strike-slip fault.

\subsection{Strain fields in the horizontal cross section}

To begin with, the effect of the free surface in the horizontal cross section is investigated. Because this effect is remarkable in the region more than $H$ away from the fault plane, the area in the present investigation is taken four times as large as that in the case of 4.1. The slip-angle is fixed to $45^{\circ}$ so that the slip vector has both dip- and strike-component.

The effect of the free surface appears clearly in the strain components such as $\partial u_{z} / \partial x$ and $\partial u_{y} / \partial z$, which constitute the shear stresses $\tau_{z x}$ and $\tau_{z y}$. For other components, this effect is considerably small. The components, $\partial u_{y} / \partial y$ and $\partial u_{y} / \partial z$, in the plane halfway between the free surface and the upper edge of the fault are presented in Figs. 9 and 10 respectively. In Fig. 9, trend of the pattern of $\partial u_{y} / \partial y$ for Model I is almost similar to that for Model II, while $\partial u_{y} / \partial z$ in Fig. 10 behaves quite differently between Model I and II, especially in the far field. The area with negative values in the region of $y / H \geq 2.0$ and that with positive values in the third quadrant of the figure appear only in Model I, corresponding to the reverse areas in the vertical planes. In the region surrounded by these two areas, the pattern appearing in Model II is found in a contracted form. Generally, difference of the strain patterns between Model I and II is small in the vicinity of the fault, where $R_{1}$ is much smaller than $R$ (see Eqs. (2.18) and (2.20)) and the main contribution to the strain field for Model I comes from $\boldsymbol{u}^{2}$ in Eq. (2.1).

Next, variation of the strain pattern with depth is investigated. In Fig. 11 , example of the strain component $\partial u_{x} / \partial y$ for three different depths is given. (a), (b), and (c) are the cases of the free surface $(z / H=0)$, halfway between the free surface and the upper edge of the fault $(z / H=\sqrt{3} / 4-7 / 10 \fallingdotseq-0.267)$ and $z / H=-0.8$ respectively. In the case (c), the fault plane intersects the plane where the pattern is given. The difference between the patterns for (a) and (b) is very small, which is also seen for other strain components. But significant differences near the fault between (b) and (c) exist. In (c), just above the fault plane, the area with negative values appearing in (b) vanishes and at the two terminals of the intersection of the fault, the contour lines diverge.

\subsection{Patterns for some invariants}

For the physical understanding of the deformation field, the invariants of strain tensor are more appropriate than the components considered above, which are dependent on the coordinates of reference.

As a first example, the dilatation in vertical cross section is given in 


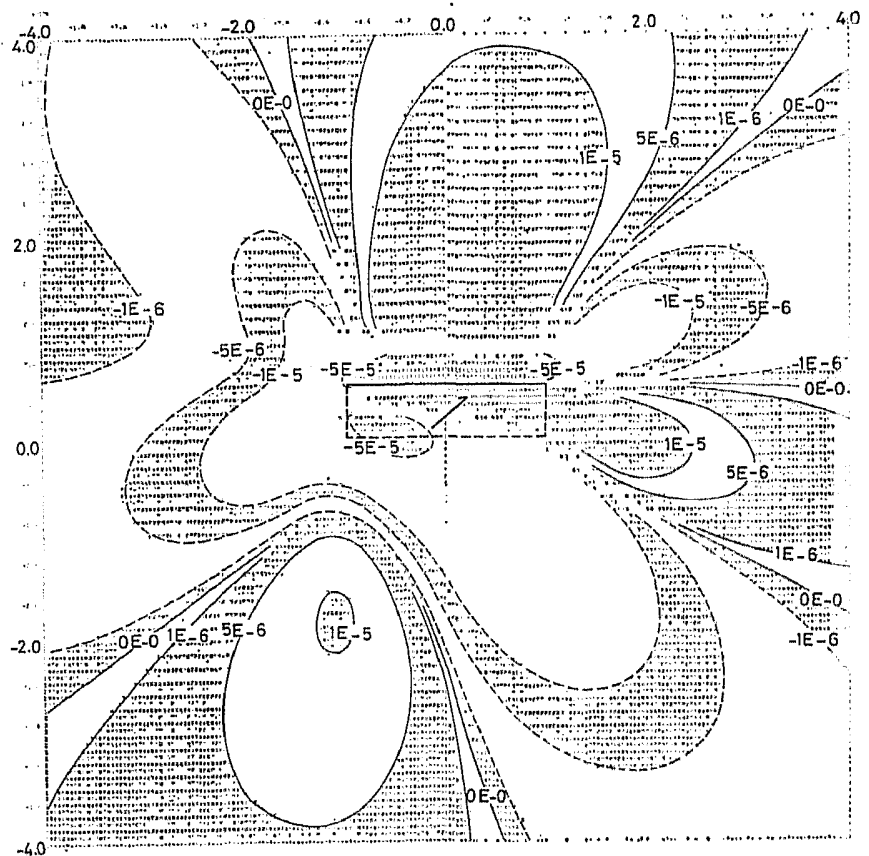

(a)

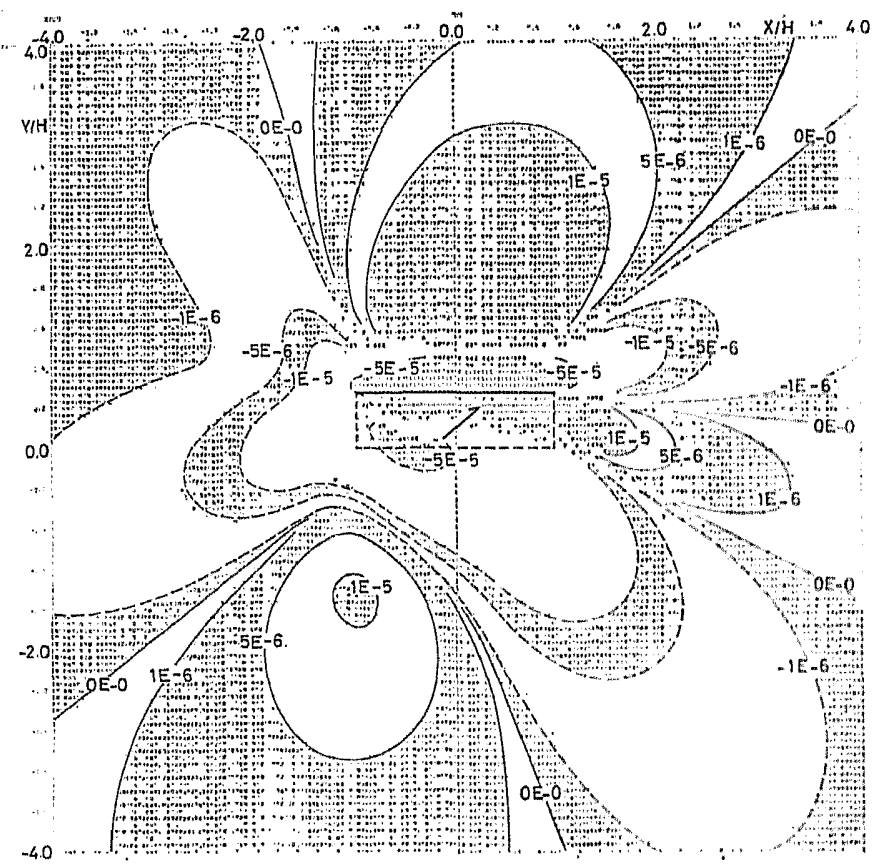

(b)

Fig. 9. $\partial u_{y} / \partial y$ in the horizontal plane halfway between the free surface and the upper fault edge $(z / H=\sqrt{3} / 4-7 / 10 \doteqdot-0.267)$ for the case of $z \cdots 45^{\circ}$. The difference between Model I and II is very small. (a) Model I, (b) Model II. 


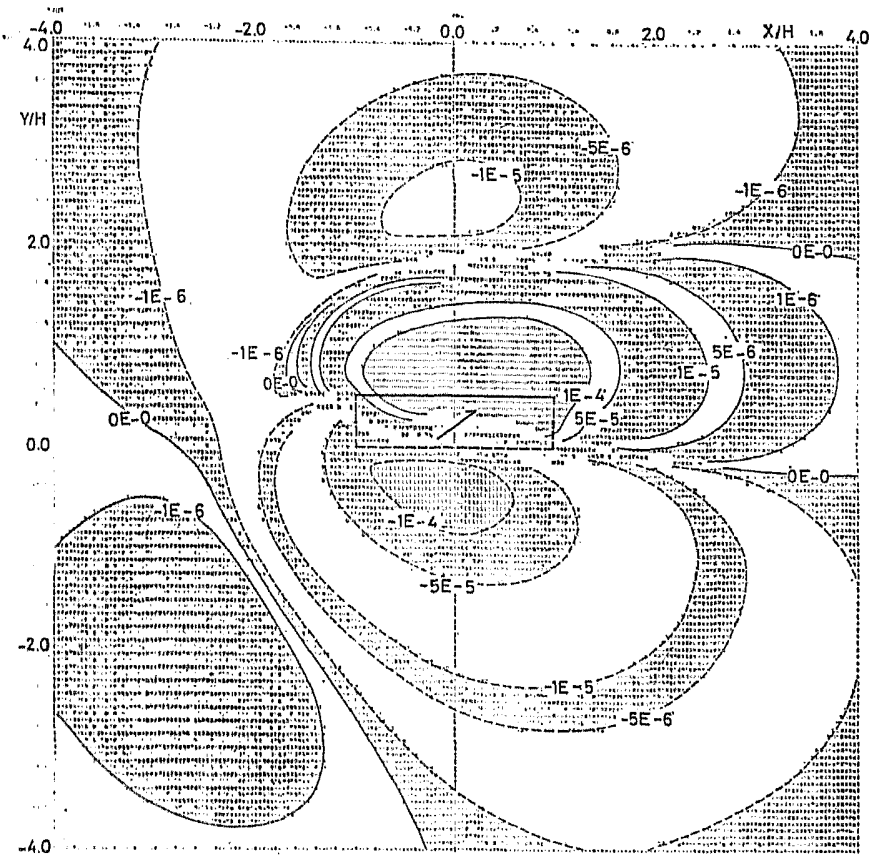

(a)

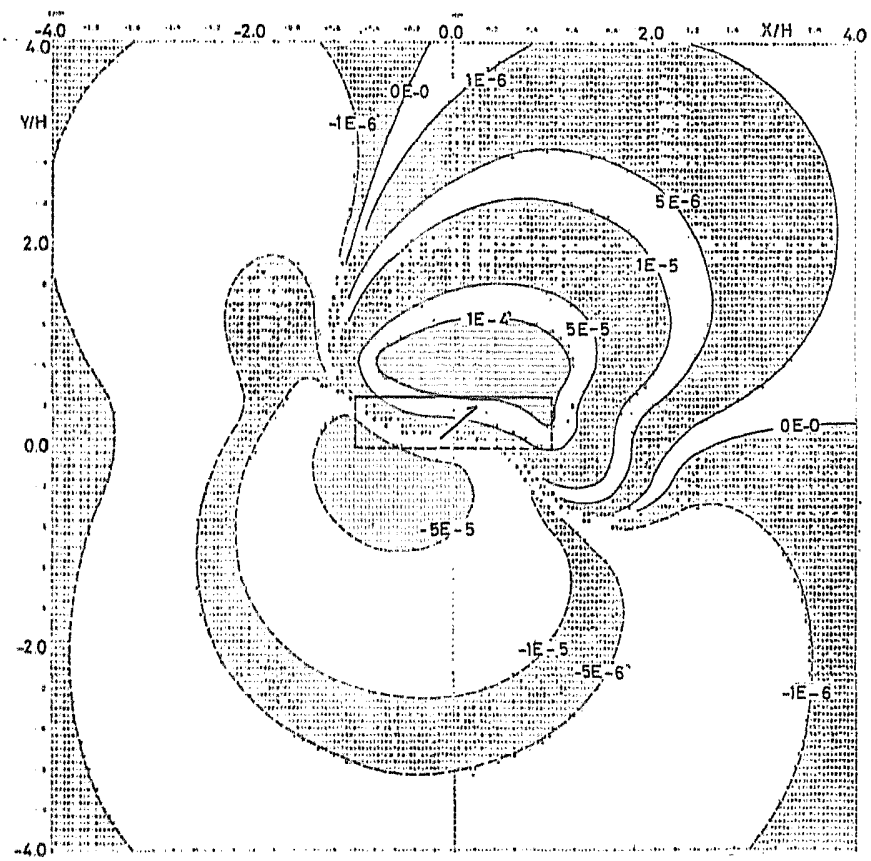

(b)

Fig. 10. $\hat{a} u_{y} / \hat{\partial} z$ in the same horizontal plane as in Fig. 9. In the regions of $y / H \geq 2.0$ and the third quadrant, the patterns of Model I and II are quite different from each other. (a) Model I, (b) Model II. 


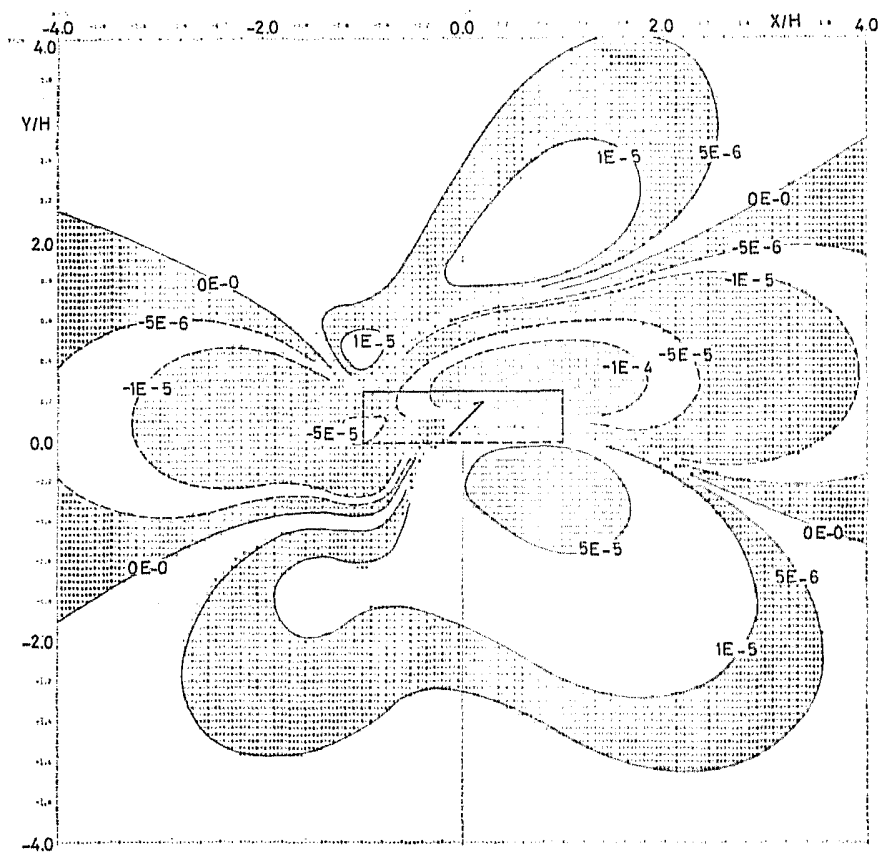

Fig. 11(a)

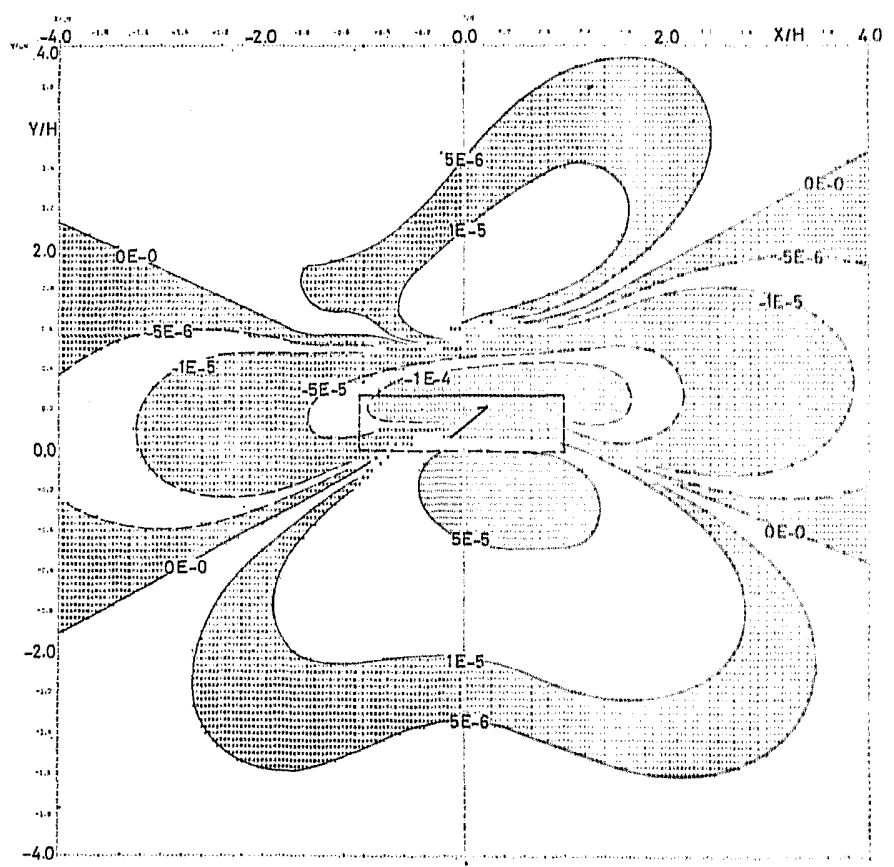

Fig. 11(b) 


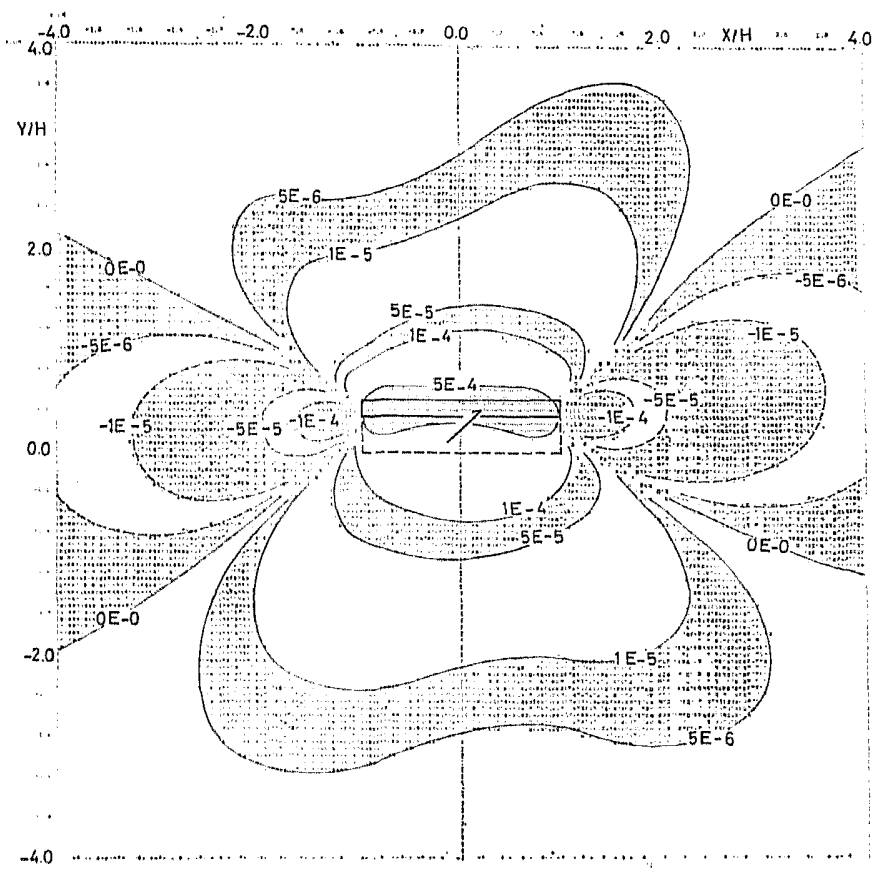

Fig. 11(c)

Fig. 11. Variation of the pattern of $\partial u_{x} / \partial y$ with depth. A remarkable difference is found between (b) and (c) in the vicinity of the fault. (a) $z=0$, (b) $z / H=$ -0.267 , (c) $z / H=-0.8$.

Fig. 12 for the pure strike- and dip-slip fault. The computation is done in the plane $(x / H=1)$ for the former case and in the $y z$ plane $(x / H=0)$ for the latter case. For the pure strike-slip fault, the medium in the upper-block side of the fault is contracted according to the fault motion and the dilatation has negative values. In the lower-block side of the fault, the dilatation is positive. Thus the pattern of the dilatation is almost anti-symmetric with respect to the fault plane in the vicinity of the fault. For the dip-slip fault, contraction and dilatation near the fault are clearly seen, which form pattern of a quadrant type. In both the cases, the patterns close to the free surface are quite different from those for an infinite medium. This difference comes from vanishment of the stress component $\tau_{z z}$ at the free surface of the semi-infinite medium.

As a second example of the invariants, the principal strains are computed in the $y z$ plane for the pure dip-slip fault (Fig. 13). The principal strains with the directions parallel to the $y z$ plane are expressed as follows;

$$
r_{ \pm}=\frac{\partial u_{y}}{\partial y}+\frac{\partial u_{z}}{\partial z} \pm \sigma, \quad \theta_{ \pm}=\tan ^{-1}\left[\left(\gamma_{ \pm}-2 \frac{\partial u_{y}}{\partial y}\right) /\left(\frac{\partial u_{y}}{\partial z}+\frac{\partial u_{z}}{\partial y}\right)\right],
$$




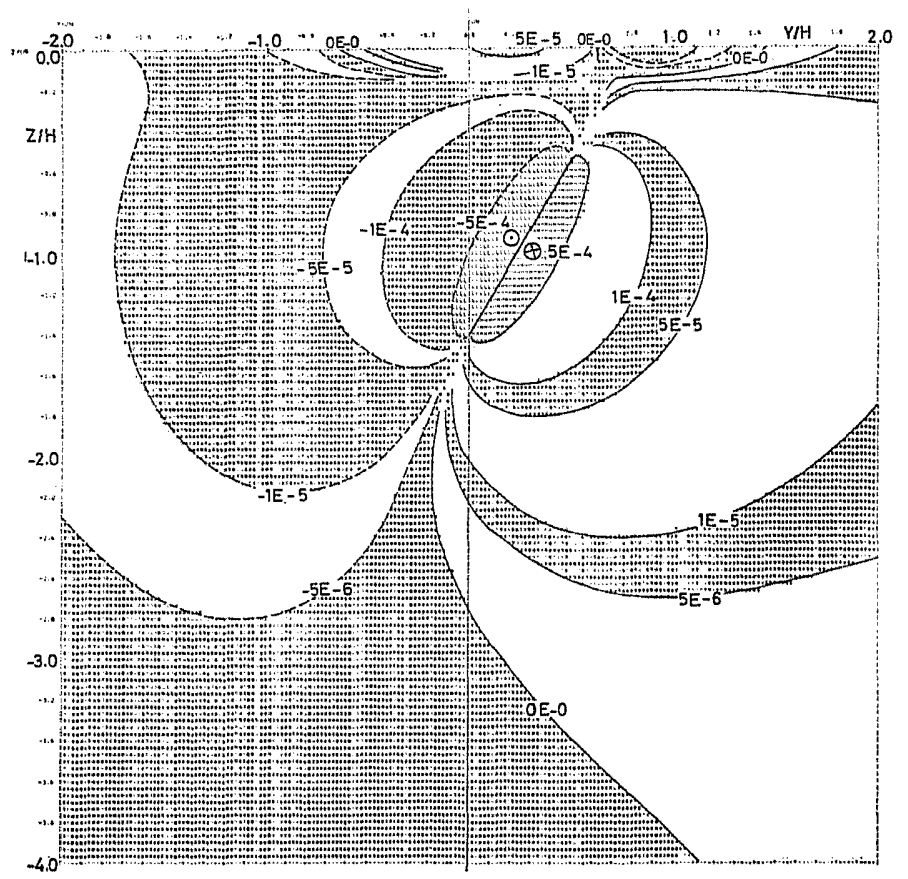

(a)

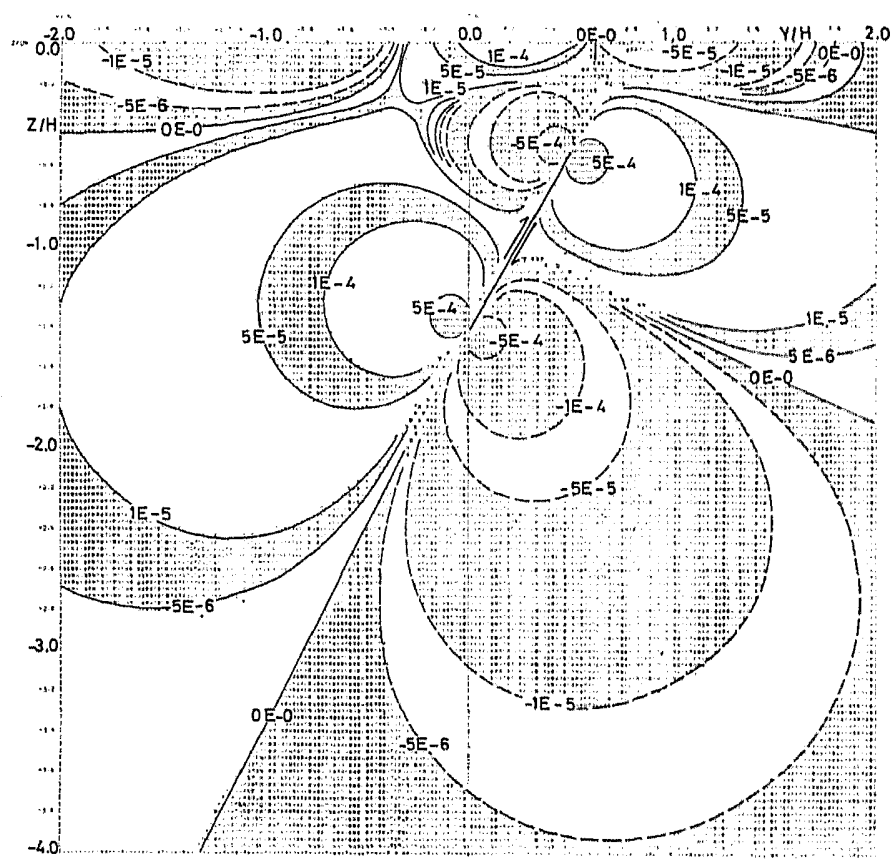

(b)

Fig. 12. Dilatations for the pure strike- and dip-slip fault. (a) $\lambda=0^{\circ}$, (b) $\lambda=90^{\circ}$. 


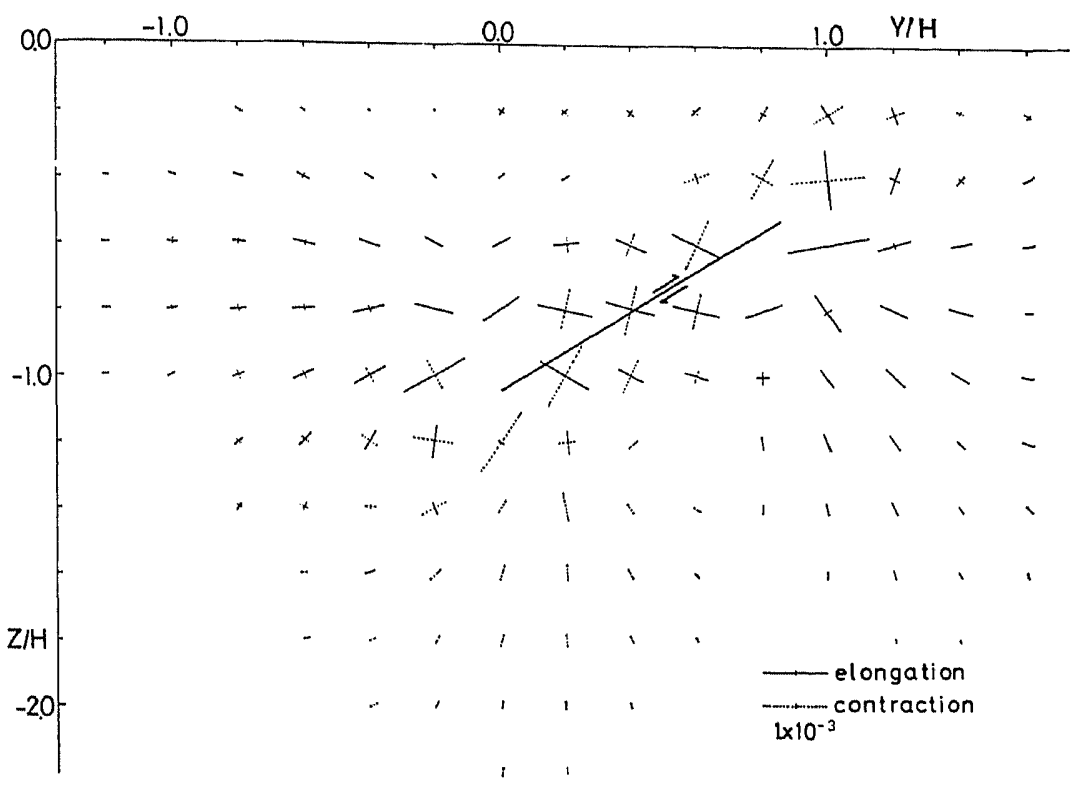

(a)

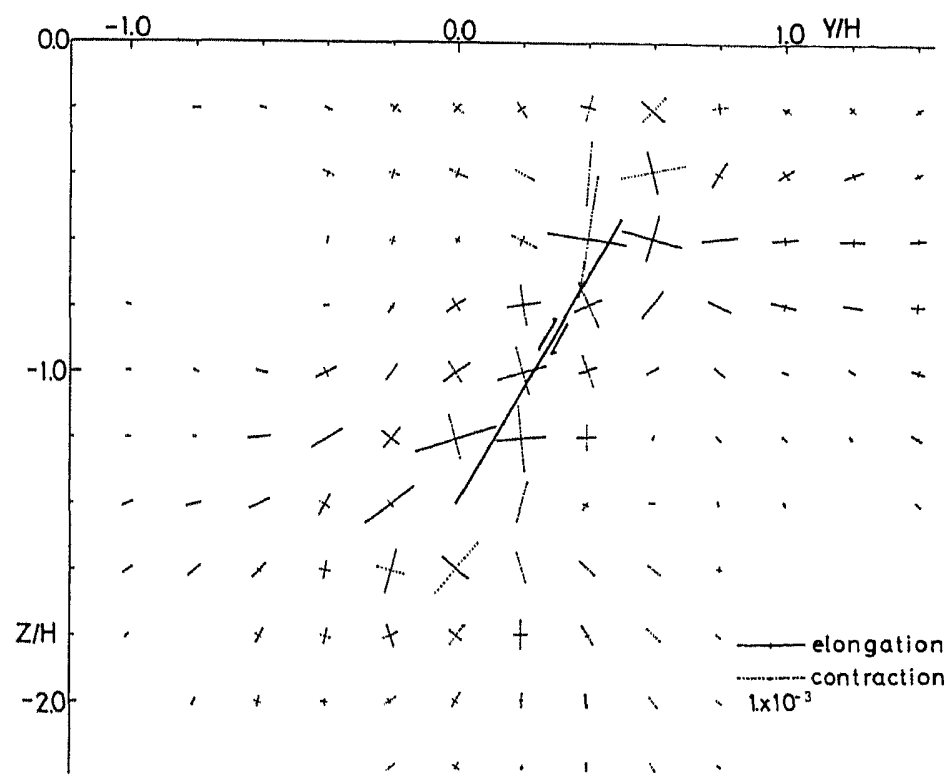

(b)

Fig. 13. The principal strains in the $y z$ plane due to the pure dip-slip fault. The depth of the upper edge of the fault for $\delta=30^{\circ}$ is the same as that for $\delta=60^{\circ}$. (a) $\delta=30^{\circ}$, (b) $\delta=60^{\circ}$. 
where

$$
\sigma=\left[\left(\frac{\partial u_{y}}{\partial y}-\frac{\partial u_{z}}{\partial z}\right)^{2}+\left(\frac{\partial u_{z}}{\partial y}+\frac{\partial u_{y}}{\partial z}\right)^{2}\right]^{1 / 2}
$$

$\theta_{ \pm}$is the angle between the $y$-axis and the principal axis of $\tau_{ \pm}$.

The computation is done in the cases of $\delta=30^{\circ}$ and $60^{\circ}$. In both the cases, magnitudes and directions of the principal strains show distribution of a quadrant type near the fault plane. In the case of $\delta=30^{\circ}$, the elongation in a direction almost parallel to the $y$-axis are predominant in the region of $-0.8 \leq z / H \leqslant-0.6$ spreading horizontally. On the other hand, in the case of $\delta=60^{\circ}$, the predominant region of elongation descends deeper with decrease of $y / H$ in the upper-block side of the fault. Hence, based upon the elastic rebound theory, if the medium is pushed and contracted in the horizontal direction, for example, the plate boundary outside an island arc, it may be expected that the earthquake occurring in that region is of a thrust type with a lower dip-angle such as $\delta=30^{\circ}$.

One more principal strain for the pure dip-slip fault, whose axis is perpendicular to the $y z$ plane, has the magnitude of $2 \partial u_{x} / \partial x$. In Fig. 14, the pattern of $\partial u_{x} / \partial x$ in the $y z$ plane is shown for $j=60^{\circ}$. Because the slip

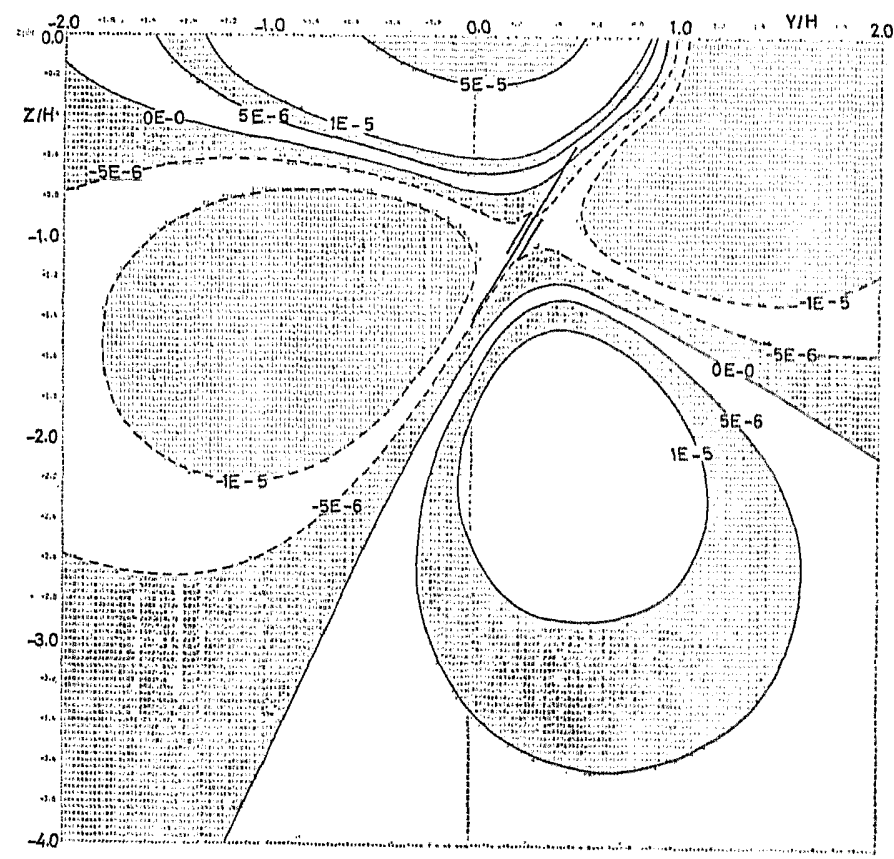

Fig. 14. $\partial u_{x} / \partial x$ in the $y z$ plane due to the pure dip-slip fault with $\delta=60^{\circ}$. Another principal strain different from those shown in Fig. 13, has the magnitude of 2. $\partial u_{x} / \partial x$ and the principal axis perpendicular to the $y z$ plane. 
motion is independent of the $x$-direction in this case, $\partial u_{x} / \partial x$ shows a very simple pattern of the quadrant type. From the results of Figs. 13 and 14, it is clear that the relation $\left|\gamma_{ \pm}\right| \geq 50 \times 2\left|\partial u_{x} / \partial x\right|$ holds everywhere. Thus, to the cause of the pure dip-slip fault the principal strain with the direction parallel to the $y z$ plane is much more important than that parallel to the $x$-axis. Considering the slip motion, this is a physically reasonable result.

\section{Conclusion}

Analytical expressions for strain field are derived for an inclined rectangular fault in a semi-infinite medium. They hold not only at the free surface but within the medium.

On the base of these expressions, a computer program for the computation of static deformation is developed and strain fields in a semi-infinite medium are investigated.

Patterns of the strain fields are much affected by the existence of the free surface. In the vertical cross sections, the effect of the free surface yields saddle points and a concentration of contour lines and also a "reverse area" where sense of deformation is opposite to that for an infinite medium. Appearance of the reverse area is remarkable in the components of $\partial u_{x} / \partial z$ and $\partial u_{y} / \partial z$. In the horizontal cross sections, the reverse area, as the effect of the free surface, appears clearly in the components which constitute the shear stresses $\tau_{x x}$ and $\tau_{z y}$.

The invariants of the strain tensor are appropriate to understanding the deformation of the medium physically. In this paper, we investigate the dilatations and the principal strains in the vertical cross-sections, whose patterns are consistent with the fault motion, especially in the vicinity of the fault plane. In the shallow region, the pattern is very complicated because of the effect of the free surface.

The computer program is available to anyone who wishes to use it for scientific purpose.

The authors are grateful to Dr. M. Matsu'ura and Mr. K. Suyehiro, Faculty of Science, University of Tokyo, who gave valuable suggestions for this work and who kindly read the manuscript. The computations were made on a HITAC 8800,8700 at the Computer Center, University of Tokyo.

\section{REFERENCES}

ALI:WINI, R. W., Application of linear inversion theory toward the estimation of seismic source parameters, Ph. D. Thesis, California Institute of Technology, Pasadena, 303 pp., 1974. 
CANITEz, N. and M. N. Toksöz, Static and dynamic study of earthquake source mechanism: San Fernand Earthquake, J. Geophys. Res., 77, 2583-2594, 1972.

Chinnery, M. A., The stress changes that accompany strike-slip faulting, Bull. Seismo!. Soc. Am., 53, 921-932, 1963.

Mansinha, L. and D. E. SMylie, The displacement fields of inclined fault, Bull. Seismol. Soc. Am., 61, 1433-1440, 1971.

PRESs, F., Displacements, strains and tilts at tele-seismic distances, J. Geophys. Res., 70, 23952412,1965 .

Sato, R. and M. MAtsu'ura, Strains and tilts on the surface of a semi-infinite medium, $J$. Phys. Earth, 22, 213-221, 1974.

Savage, J. C. and L. M. HAstie, Surface deformation associated with dip-slip faulting, $J$. Geophys. Res., 71, 4897-4904, 1966.

Steketee, J. A., On Volterra's dislocations in a semi-infinite medium, Carl. J. Phys., 36, 192-205, 1958 a.

Steketee, J. A., Some geophysical applications of the elasticity theory of dislocations, Can. J. Phys., 36, 1168-1198, 1958 b. 